\title{
Disjoint combinations profiling (DCP): a new method for the prediction of antibody CDR conformation from sequence
}

The accurate prediction of the conformation of Complementarity-Determining Regions (CDRs) is important in modelling antibodies for protein engineering applications. Specifically, the Canonical paradigm has proved successful in predicting the CDR conformation in antibody variable regions. It relies on canonical templates which detail allowed residues at key positions in the variable region framework or in the CDR itself for 5 of the 6 CDRs. While no templates have as yet been defined for the hypervariable CDR-H3, instead, reliable sequence rules have been devised for predicting the base of the CDR-H3 loop. Here a new method termed Disjoint Combinations Profiling (DCP) is presented, which contributes a considerable advance in the prediction of CDR conformations. This novel method is explained and compared with canonical templates and sequence rules in a 3-way blind prediction. DCP achieved $93 \%$ accuracy over 951 blind predictions and showed an improvement in cumulative accuracy compared to predictions with canonical templates or sequence-rules. In addition to its overall improvement in prediction accuracy, it is suggested that DCP is open to better implementations in the future and that it can improve as more antibody structures are deposited in the databank. In contrast, it is argued that canonical templates and sequence rules may have reached their peak. 


\section{Disjoint combinations profiling (DCP): a new method for the}

2 prediction of antibody CDR conformation from sequence.

3 Dimitris Nikoloudis ${ }^{1}$, Jim E. Pitts ${ }^{1}$ and José W. Saldanha ${ }^{2 *}$

4

$5 \quad$ I'Department of Biological Sciences, Birkbeck College, University of London, Malet Street,

6 London, WCIE 7HX, UK

7

$8 \quad{ }^{2}$ Division of Mathematical Biology, National Institute for Medical Research, The Ridgeway,

9 London NW7 1AA, UK

10

$11 *$ corresponding author

12 Email: Email: jsaldan@nimr.mrc.ac.uk

13

14 Tel: (+44)(0) 2088162299

15 Fax: (+44)(0) 2088162460

16

17

18

19

20

21

22

23

24 


\section{ABSTRACT}

28 The accurate prediction of the conformation of Complementarity-Determining Regions (CDRs) is 29 important in modelling antibodies for protein engineering applications. Specifically, the 30 Canonical paradigm has proved successful in predicting the CDR conformation in antibody 31 variable regions. It relies on canonical templates which detail allowed residues at key positions in 32 the variable region framework or in the CDR itself for 5 of the 6 CDRs. While no templates have 33 as yet been defined for the hypervariable CDR-H3, instead, reliable sequence rules have been 34 devised for predicting the base of the CDR-H3 loop. Here a new method termed Disjoint 35 Combinations Profiling (DCP) is presented, which contributes a considerable advance in the 36 prediction of CDR conformations. This novel method is explained and compared with canonical 37 templates and sequence rules in a 3-way blind prediction. DCP achieved 93\% accuracy over 951 38 blind predictions and showed an improvement in cumulative accuracy compared to predictions 39 with canonical templates or sequence rules. In addition to its overall improvement in prediction 40 accuracy, it is suggested that DCP is open to better implementations in the future and that it can 41 improve as more antibody structures are deposited in the databank. In contrast, it is argued that 42 canonical templates and sequence rules may have reached their peak. 


\section{${ }_{54}$ Introduction}

55

Antibodies can recognise virtually any given molecule mainly by variation in the length and sequence of their Complementarity-Determining Regions (CDRs), which form the antibody's binding interface. Three CDRs are found in the antibody's Heavy chain (CDR-H1, -H2, -H3) and three in the Light chain (CDR-L1, -L2, -L3). The first definition of CDRs was by Wu \& Kabat (1970) while performing an analysis of the variable domains of Bence-Jones proteins and myeloma Light chains. Later, Kabat and colleagues compared the sequences of the hypervariable regions in the then known structures and observed that at 13 sites in the Light and 7 in the Heavy chains (Kabat, Wu \& Bilofsky, 1977), the residues are conserved. They suggested that these positions in the sequence are involved with structure rather than specificity, introducing for the first time a possible relationship between sequence and loop conformation in antibodies. A second set of observations of the crystal structures of Fab fragments and myeloma proteins revealed that, in many cases, hypervariable regions with the same length but different sequences have the same main chain conformation (de la Paz et al., 1986).

It was in 1986 (Chothia et al., 1986) that specific residues were directly associated with the conformation of the hypervariable regions during a visual analysis of the sequence and structure of antibody D1.3, thus introducing the notion of the "canonical model". From this point, various further studies enriched the table of structurally-determining residues (canonical residues), by observing the amino acid similarities at key interacting positions within sequences of members of any given conformational class, of the known and newly defined canonical structures, for the three CDRs in Light and the first two in Heavy chains (Chothia \& Lesk, 1987; Chothia et al., 1989; 1992; Barré et al., 1994; Tomlinson et al., 1995; Guarne et al., 1996; Martin \& Thornton, 1996; Morea, Lesk \& Tramontano, 2000; Vargas-Madrazo \& Paz-Garcia, 2002). Therefore, these collections of structurally-determining residues created canonical templates for each known conformational class, which defined the allowed residues per identified position in the variable chain. These canonical templates could then be used for prediction, from sequence alone, of the conformation of a new CDR by requiring its variable chains match as many, if not all, of the allowed residues present in the template. Regarding the sixth and final CDR-H3, a number of studies (Shirai, Kidera \& Nakamura, 1996; 1999; Furukawa et al., 2001; Kuroda et al., 
84 2008) provided structure-determining sequence rules for the prediction of the CDR-H3-base (or

85 'take-off', 'torso' or 'anchor') conformation.

86 In the latest relevant study (North, Lehmann \& Dunbrack, 2011), it was inferred that the 87 effect of canonical residue overlap between templates caused by the proliferation of structures 88 was diminishing the efficacy of the canonical model. Instead, a mixed approach was proposed for 89 prediction of CDR conformation, sometimes based on the presence of a very small number of statistically prominent structurally-determining residues, the gene source, CDR length or even the use of Hidden Markov Models (HMMs). Therefore most conformational clusters/classes were noted as not canonical, while a considerable number were characterised as non-predictable altogether. Furthermore, concerns were raised regarding the predictability from sequence of the bulged (including double-bulged) CDR-H3-base conformation.

The accurate prediction of CDR conformation is important in modelling antibodies for protein engineering applications (e.g. ab initio design of antibodies, antibody humanisation, vaccine design, etc.). Specifically, knowledge of the CDR conformation is crucial for the creation of a stable binding interface, modification of the antibody's binding affinity or even identification of an epitope. Computational methods such as the canonical model or CDR-H3 sequence rules, which attempt conformational prediction of CDRs from sequence alone, have the advantage of being inexpensive and fast while requiring only a simple input; their major drawback being the inability to predict conformations that were never observed before experimentally. In this context,

103 a re-evaluation of the performance of the canonical model in predicting the class of CDR 104 conformation from sequence alone is presented in light of the latest new and multi-level complete 105 CDR clustering (Nikoloudis, Pitts \& Saldanha, 2014, PeerJ preprint server, DOI: 106 10.7287/peerj.preprints.291). The key residues are updated in the existing canonical templates 107 from the sequences of members of each level-1 cluster/class, and correspondingly the canonical 108 templates for new clusters in a given length are populated, using the key positions defined for that length by Martin \& Thornton (1996). Those defined key positions are identical for all clusters of

110 a given length. In this way, an assessment as to whether the canonical model is still effective as 111 the quickest and simplest prediction method for antibody CDR conformation is carried out, and 112 the effect of canonical residues' overlap between templates caused by the proliferation of cluster 113 sequence populations can be evaluated.

114 For the hypervariable (both in sequence and conformation) CDR-H3, the sequence rules 115 for CDR-H3-base prediction described in Shirai, Kidera \& Nakamura (1999) are tested, as well 116 as their updated versions in Kuroda et al., (2008). The goal here is to compare the accuracy of the 
117 two sets of rules and, more importantly, to find out if the continual adaptation to new sequences

118 with additional rules, exceptions and overrides is beneficial to this predictive model.

119 Besides testing these two popular and historic approaches on an updated dataset, a new 120 predictive model from sequence alone is also introduced which aims to bring improved accuracy 121 over previous sequence-based methods, while retaining their rapid execution and simplicity of 122 usage. All the characteristics of the new method are detailed, step-by-step: inception, goals, basic 123 concepts and definitions, implementation strategies, training and prediction workflows. A 124 demonstration is presented of a standard predictive model derived from the method as well as an 125 assessment of its efficacy on the same set of CDRs employed for the testing of the canonical 126 model and CDR-H3-base rules. As this new method allows parameterisation, future dedicated 127 work could take advantage of the general framework provided and propose a number of different 128 or improved implementations.

129 The prediction results obtained by the new method are directly compared to those from 130 previous approaches, and complemented by statistical characteristics of the training, validation 131 and test sets. Additionally, special importance is attributed to each method's performance in 132 predicting the major cluster/conformation (class-I) in any given CDR/length combination (e.g. 133 CDR-L1 11-residues). Indeed, as is revealed by the population percentages per cluster in 134 Nikoloudis, Pitts \& Saldanha, 2014 (PeerJ preprint server, DOI: 10.7287/peerj.preprints.291), in 135 each CDR/length with more than 10 unique sequences there is usually a single cluster which 136 regroups the large majority of the known conformations, while the remaining fraction may be 137 populating a considerable number of much smaller clusters. In the 15 lengths (first 5 CDRs) that 138 contained more than 10 unique sequences in their clustered population and produced more than 139 one cluster, the major cluster of each length represented on average $74 \%$ of the available unique 140 sequences (median: 86\%). As a consequence, these major conformations are expected to occur 141 more frequently and are accordingly more probable to prove of interest in research scenarios. For 142 this reason further analysis is undertaken of the prediction results to calculate the precision, recall 143 and F-measure for all major clusters, and the corresponding comparisons between methods are 144 presented. 


\section{A new blind dataset}

As the clustering dataset in Nikoloudis, Pitts \& Saldanha, 2014 (PeerJ preprint server, DOI: 10.7287/peerj.preprints.291) was locked on the 31/12/2011 edition of the PDB (Berman et al 2000), this presented an opportunity to conduct a true blind-testing by downloading the antibody structures that were released subsequently. Hence for the new dataset, a search was performed in the PDB for structures released between 01/01/2012-21/11/2013, using the same methodology as in Nikoloudis, Pitts \& Saldanha, 2014 (PeerJ preprint server, DOI: 10.7287/peerj.preprints.291), which returned 312 files, two of which contained structures from 3 antibodies (PDB codes 3ULU, 3ULV). After removing redundant sequences, there remained a total of 230 antibody structures: 210 had both Heavy and Light chains, 4 had only a Light chain and 16 had only a Heavy chain. All redundant instances (i.e. multiple copies of the same CDR sequence within the same structure and CDRs from different structure files with identical Fv sequences) were additionally searched for different CDR conformations. Only one of the 230 structures was retained despite the fact that it was redundant (4DN4), because a different CDRversions, respectively).

As DCP required parameter tuning, a validation step had to be inserted. However, since the initial structure of the data to be predicted presented a majority of clusters with only between one to three unique sequences, it proved impractical to perform a traditional k-fold crossvalidation on the clustered set as these smaller clusters could not be further subdivided in a

173 meaningful way. Instead a 3-way experiment was designed, where the previously clustered 174 dataset was used for training, while the new dataset was divided approximately in half into a 175 validation set and a test set. The validation set comprised of all PDB files released between 176 01/01/2012 and 14/03/2013 (113 non-redundant antibody structures), while the test set included 177 all the subsequently released structures (15/03/2013 to 21/11/2013, 117 non-redundant antibody 178 structures). This division of the dataset by time preserved the double-blind nature of the 179 experiments, since the complete test dataset was also constructed with time of release as the sole 180 criterion, thus eliminating any subjectivity from the selection, analysis and interpretation. 
An examination of the redundant sequence content (complete Fv identity) between

182 training and test datasets was also performed. This count revealed a 7\%-9\% fully redundant

183 sequence content in the test dataset (i.e. present in the training dataset) in all considered CDRs

184 (specifically, full count[subset-1 count/subset-2 count]: 11[4/7], 13[7/6], 17[7/10], 17[7/10] and

185 17[7/10], for CDR-L1, CDR-L3, CDR-H1, CDR-H2, CDR-H3-base, respectively, supplementary

186 material 'individual_CDR_predictions.xls'). While the fully redundant content appeared to be 187 relatively low, the concerned entries were retained in the test dataset in order to allow an 188 appreciation of the methods' accuracy in predicting a trained sequence and demonstrate their 189 capacity to overcome overlapping predictive definitions.

190 By using this new dataset, it was possible to retain the previous entire clustered set as a 191 prior knowledgebase and to assess the sequence-based prediction methods in realistic conditions 192 without discarding or ignoring any data, both during training/updating and testing. This ensured 193 that DCP training and canonical templates' updating remained blind toward the new PDB files. In 194 terms of predictions with canonical templates, the entire new dataset served for testing since no 195 validation step was required. However, for practical reasons, the above first subset will 196 henceforth be called "the validation set" (for DCP) and the second subset "the test set" (for DCP), 197 despite the fact that both constitute test sets for the canonical method.

198 All conformational predictions were applied at the first level of the clustering set's nested 199 scheme. New Fv sequences were numbered, using the numbering scheme and CDR extents 200 described in Nikoloudis, Pitts \& Saldanha, 2014 (PeerJ preprint server, DOI: 201 10.7287/peerj.preprints.291). The Ca-backbones of new CDRs were then successively 202 superposed onto the medoid structure of every cluster of the same length, in order to determine 203 the actual conformation of new CDRs. For a new CDR to be assigned to a pre-existing 204 conformational cluster, its RMSD to the cluster's medoid was required to be lower than the 205 cluster's radius.

\section{A new method for prediction of CDR conformation from sequence}

\section{Method presentation}

It has been made clear through various studies (Chothia et al., 1989; 1992; Alzari et al.,

213 1990; Al-Lazikani, Lesk \& Chothia, 1997; Martin \& Thornton, 1996; Morea, Lesk \& 
214 Tramontano, 2000; Vargas-Madrazo \& Paz-Garcia, 2002; Shirai, Kidera \& Nakamura, 1996;

215 1999; Kuroda et al., 2008) that the CDR sequence is not always solely determinant of the CDR

216 conformation. Several residues external to the CDR, from the framework, other CDRs or the

217 second Fv chain, were retained as structurally-determinant and included in predictive canonical

218 templates or sequence rules. These residues were spotted after pedantic visual examination of a

219 number of antibody structures of interest, as making important contacts with CDR residues.

220 However, this process can potentially lead to misleading generalisations due to crystal errors, or

221 the intrinsic backbone and side-chain flexibility of surface residues such as those in CDR 222 sequences.

223 In the new method now presented, a generalisation for the presence of class-specific 224 combinations of residues is proposed. These combinations of residues would represent 225 conformation-influencing synergies that are expected to appear exclusively or preferentially in 226 members of one cluster. As far as the physico-chemical aspect of the residues' interaction is 227 concerned, these combinations may be representing steric effects, creation of a hydrophobic 228 pocket or local environment, hydrogen-bonding, van der Waals' contacts, salt bridges, backbone 229 flexibilities, etc. Of course any investigation of sequence sets with such physico-chemical criteria 230 would dramatically increase the complexity of any method. Instead a simpler model is proposed 231 where the nature of these interactions, as well as the very residues which participate, remain 232 irrelevant to the prediction procedure. More specifically, it would be of interest to search for 233 those combinations of positions in the antibody Fv sequences that contain combinations of 234 residues that are always different between different conformational clusters, i.e. combinations of 235 positions that present disjoint combinations of residues between classes. In this way the sequence 236 differences between different classes are examined, instead of the sequence similarities within a 237 class as is the case with the canonical model. This approach was named 'Disjoint Combinations 238 Profiling', or DCP, and all its characteristics are further detailed in the following sections.

\section{Basic definitions}

For the formulation of this new method a number of novel features needed to be defined, 243 which are detailed later. The basic terms used in the DCP prediction method are provided here in 244 a table (Table 1), as both an introduction and for quick reference. 
In this demonstration of DCP, all neighbouring residues of a CDR are included, within a radius of $4 \AA, 6 \AA$ or $8 \AA$, as potentially interacting with the CDR in a way that is influencing its conformation. The initial assumption is that these neighbourhoods of members of the same conformational cluster have equivalent influence on the observed conformation. Therefore, it is expected that within these neighbourhoods there exist combinations of positions that make distinct conformational-influencing synergies, and whose sequences are never observed in members of a different cluster. These synergies could be caused by any number of the aforementioned residue-to-residue interactions. The theoretical basis behind this parameter could be the chained influence that residues may have on a local conformational feature, also implicating residues that make indirect contact with the CDR; e.g. a cascade of interactions between 3 or 4 residues where the last residue resides on the CDR but makes no contact whatsoever with the first residue of the cascade. It is therefore possible that DCP captures such chained synergies, which are different between different conformational classes.

All the Fv positions that are predominantly found within the selected radius of an examined CDR, its residues included, define its 'Interaction Frame' (IF). This frame of positions was constructed after visual examination with the graphics program Swiss-PdbViewer (Spdbv; Guex \& Peitsch, 1997) of a large number of antibody structures. During visual examination, all positions that satisfied the radius criterion and were common to all members of all clusters, were retained. As the antibody framework is very stable, the vast majority of neighbouring positions that were observed (over 90\%) was topologically preserved between the examined CDRs. This operation was repeated for each CDR.

Once the IF is selected for a given CDR, the sequences of all cluster members per CDR/length combination are parsed for the residues that occupy the Fv positions found in the IF. These residues are then arranged in the same order as the respective positions appear in the IF, in order to form the corresponding 'IF sequence'. This way, each cluster now has a set of IF

273 sequences that can be compared with each other for the detection of disjoint combinations of 274 residues between them. A graphical representation of these setting-up steps can be seen in Figure 2751.

A common problem in CDR conformational prediction from sequence alone is the presence of sparsely populated clusters/classes. The sequence examples of those clusters are often

278 so few that it becomes impossible to detect sequence features that are at the same time common 279 between members of that cluster but different from other clusters. Especially so when the major 
cluster in a given length also has few members; any comparisons between the different clusters' sequences become prohibitively risky. For the DCP training process, this obstacle was overcome

282 by regrouping the sequences of all clusters in that length, except for the one that is being profiled.

283 Indeed, in searching for differences, the profiled class needs to be presented against an 'anti284 reference' rather than a traditional 'reference' used in many prediction methods. For example, it is possible to screen class A against what "is_not_class_A", so by regrouping all "non_class_A" instances there is a practical enrichment of the volume of sets of sequences to be compared.

The 'Query IF sequence set' was defined as the group of non-redundant IF sequences of all members of the cluster under examination and the 'Target IF sequence set' was defined as a group of non-redundant IF sequences from members of all clusters except for the one that is being profiled. For example, when examining cluster-1 in a CDR/length with 4 clusters, a comparison is made of Query IF sequence set [cluster-1] versus Target IF sequence set [clusters2/3/4]. The profiling for disjoint combinations can then be initiated by cycling through all combinations of Fv positions within the IF, up to the maximum combinatorial order that is preselected (e.g. singlets, couplets, triplets, quadruplets, or quintets, etc., each time including combinations of lower order), and extracting the corresponding amino acid sequences from the Query/Target IF sequence sets. Each combination of positions was called an 'IF fragment' and, accordingly, the corresponding extracted residues formed an 'IF fragment sequence'.

Once all respective amino acid fragment sequences are acquired from both Query and Target sets, the corresponding fragment sets are then examined for disjointness, i.e. that no sequence fragment is shared between the two sets. If the sets prove to be disjoint, that IF fragment is retained as pointing to a potentially significant difference between the two sets. This IF fragment is called a 'Signature signal'. The rationale is that if any sequence combination of the examined IF fragment is shared even once between the members of the different clusters, then the examined IF fragment sequences are not mutually exclusive and therefore cannot be theoretically considered as unique to any conformation. The complete list of signature signals constitutes the 'DCP signature' of the examined (Query) cluster/class, which is consequently used for its prediction with new sequences. A graphic representation of this training process can be seen in 308 Figure 2.

As a note, the basic properties of combinations imply that the observance of any signature signal of lower order automatically renders equally disjoint any combination of greater order,

311 which contains all the IF positions of the lower order combination. For example, when IF 312 fragment L90-L95 is disjoint, thus becoming a signature signal, any higher order combinations 
313 containing the previous IF positions are also disjoint; e.g. L90-L91-L95, L89-L90-L95, L89-L90-

314 L91-L95, etc. are all equally signature signals. Therefore, in order to avoid unnecessary

315 redundancies within a DCP signature which may affect prediction scoring, a filtering is

316 performed that removes signature signals from the DCP signature when they contain other signals

317 of lower order.

318

319 Prediction of CDR conformation with DCP signatures

320

321

Once a DCP signature and a Target IF sequence set are acquired for each conformational 322 class, it becomes possible to predict the unknown conformation of CDRs (from new Fv 323 sequences) by scoring the differences (disjoint combinations). New Fv sequences will henceforth 324 be referred to as "Query" sequences, as they become the profiled object. The first step is, again, 325 to number the Query Fv sequence and to assemble the respective IF sequence for each CDR to be 326 predicted from the residues that correspond to the IF positions (defined previously during 327 training). Subsequently, the DCP signature and the corresponding Target IF sequence set for each 328 class of the corresponding CDR/length are loaded in turn. For each screened class, the signature 329 signals are read one-by-one and the corresponding sets of IF fragment sequences are re330 constructed. These sets of Target IF fragment sequences are then examined for disjointness versus 331 the corresponding Query IF fragment sequence from the unknown CDR. If disjointness is 332 observed between the Query fragment sequence and the Target fragment sequences in a given IF 333 fragment (i.e. the Query fragment sequence is not in the list of Target fragment sequences), then 334 the comparison score is increased by 1 and comparisons proceed with the next signature signal 335 until all comparisons are performed. It is important to note again that signal matching is achieved 336 by observing sequence differences (i.e. disjoint fragments) and not sequence similarities as is 337 more common in the canonical model.

338 The final signature matching score $\left(\mathrm{R}_{\mathrm{DCPs} \text { signature }}\right)$ of a given class is equal to the comparison 339 score (total number of disjoint signals), divided by the total number of signature signals in the 340 DCP signature:

341

$342 \quad R_{D C \text { signature }}=\frac{\text { disjo } \int \text { signals }}{\text { total signature signals }}$ 
344 Once all classes in the given CDR/length are scored, the predicted conformation is the one with

345 the $\mathrm{R}_{\mathrm{DCP} \text { signature }}$ ratio closest to 1 , and the workflow is repeated for the next CDR conformation to

346 be predicted. A representation of the prediction workflow by DCP signatures can be seen in 347 Figure 3.

\section{Canonical templates}

The canonical templates were derived for every applicable conformational cluster, using the definitions of structurally-determining residues described in Martin \& Thornton, (1996). This choice was guided by the fact that the aforementioned study remains the most extended work on canonical residues, providing detailed tables of canonical templates for each conformational class.

Table 2 shows the canonical positions used for the creation of predictive templates in each applicable cluster, while the detailed canonical templates employed during blind-testing can be consulted in supplementary material: 'all_Canonical_Templates.doc'. These templates were derived from the exact same training sequences used during DCP training, in order to allow a straight comparison between the two methods. It can be argued, that due to the nature of the level-1 clusters produced in Nikoloudis, Pitts \& Saldanha (2014, PeerJ preprint server, DOI: 10.7287/peerj.preprints.291), the respective canonical templates may contain an unwarranted number of allowed residues, leading to misclassifications. This eventuality was explored by concurrently constructing, in selected cases (e.g. CDR-L3/9-residues, CDR-H1/13-residues), canonical templates from a small centralised portion of the cluster's population, where conformation variations are minimal; namely those members that belonged to the cluster's core. However, this training restriction led to an increased rate of misclassifications by canonical templates, probably because the sets of allowed canonical residues were not rich enough. For both this reason and for complete training conformity between the two methods, the exact same training sequences were used for DCP and canonical prediction from sequence. 
Two sets of sequence rules for the prediction of the CDR-H3-base conformation were

376 used: the first set from Shirai, Kidera \& Nakamura (1999) and the updated set from Kuroda et al.,

377 (2008). The second set is an extension of the original set of rules based on examination of 314

378 new, non-redundant structures from the PDB. Blind-testing both sets of rules on the available test

379 sets presented a good opportunity to examine their validity and, importantly, assess their

380 extensibility by constant adaptation to new sequence findings. Although the respective 381 publication was made in 2008, the updated set is referred to as "H3-rules 2007" in the 382 corresponding text, so will henceforth be referred to accordingly.

\section{Identification of multi-conformation full-rogue CDRs} identical CDR sequences were defined as 'rogue'. For the DCP training and construction of canonical templates, it was also essential to search for, and deal with, structures that have the exact same Light and Heavy chain sequences within the clustering (training) dataset, but contain a CDR that belongs to different conformational clusters. These CDR structures were named 'multi-conformation full-rogue CDRs'. Indeed, the presence of such CDRs in the training set would void DCP, as it would no longer be possible to detect any disjoint combinations between the sequences of the affected clusters. To a lesser degree, the same event would be detrimental for canonical predictions as well, since these full-rogue CDRs would have rogue templates, in the sense employed by Martin \& Thornton (1996). However, as noted in North, Lehmann \& Dunbrack (2011) and also observable in the detailed updated canonical templates (see Results section), the constantly increasing number of new antibody structures is already transforming most canonical templates into a 'rogue' status.

A visual examination of all detected occurrences was performed and detailed observations for Light and Heavy chain CDRs, and CDR-H3-base can be found in supplementary material: 'all_Rogue_Members.doc'. Based on these findings, it was decided to make no arbitrary exclusion of CDRs from the training set. The reason was that many rogue cases could warrant a dedicated study in order to make inferences on structure validity or potential conformational switches due to antigen/ligand contacts or backbone flexibility. Instead, it was decided that the affected clusters be merged into a combination of predictable conformations. In other words, affected clusters were treated as one during training for DCP and derivation of canonical 
templates. The implications of this choice are debated in the Discussion section. Finally, this

408 identification of multi-conformational full-rogue members is presented as a piece of subsequent 409 analysis based on the results of the complete clustering performed in Nikoloudis, Pitts \& 410 Saldanha (2014, PeerJ preprint server, DOI: 10.7287/peerj.preprints.291).

\section{Validation of DCP training parameters}

The DCP method allows selection of the CDR neighbourhood radius (IF) and the maximum combinatorial order of IF fragments. In this demonstration, IF radii of $4 \AA, 6 \AA$ and $8 \AA$

417 (3 possible selections) were considered, as well as maximum orders up to triplets and up to 418 quadruplets (2 possible selections). Therefore, DCP training per CDR/length was repeated for all 6 combinations of parameters and validated each time on the validation set. The combination of parameters that resulted in the higher predictive accuracy was retained for the final evaluation of the method on the test set. For the prediction of the CDR-H3-base conformation, quintets were also considered resulting in 3 additional training sessions. The selected parameters are listed in the Results section.

Blind-testing of sequence-based prediction methods for CDR

\section{conformation}

Prediction results were categorised into four types: accurate, uncertain, false predictions and novel conformations. Predictions were considered failed in all cases other than the category "accurate". As the prediction result from DCP signatures and canonical templates is based on the ratio of matched over the total number of signals/canonical residues, it is possible for two conformational classes to obtain the same maximum score. In these cases, the prediction is

435 'uncertain', and all classes with identical maximum score are output for reference. For an 436 accurate prediction, the RMSD distance of the examined CDR conformation from a single 437 cluster's medoid was required to fall within that cluster's radius. If this requirement was not 
438 matched, then the conformation was considered novel. In a few cases, the examined

439 conformation appeared as an outlier between two clusters, displaying very similar RMSD

440 distances to both their medoids; these outliers were also considered as novel conformations.

441 Conformations with a CDR length with only one available cluster did not count towards any 442 evaluation.

443 For the assessment of each method's performance with regard to the prediction of the 444 major cluster (class-I) in each CDR/length, the following measures are calculated:

445

446

$$
\text { Accuracy }=\frac{T P+T N}{T P+T N+F P+F N}
$$

447

$448 \quad$ Precision $=\frac{T P}{T P+F P}$

449

$450 \quad \mathfrak{R}$ call $=\frac{T P}{T P+F N}$

with TP: True Positive, TN: True Negative, FP: False Positive, FN: False Negative. Here, the 453 positive class is the major conformation and the negative class refers to all the other conformations in that length. Therefore, 'True Negative' refers to the accurate prediction of a conformation other than the major in that length. Accordingly, 'False Negative' refers to the false prediction of a conformation other than the major one in the given length, while the actual conformation is the positive class.

458 Finally, as a technical appreciation of the combination of precision and recall, the F-measure is also provided:

460

$461 \quad F=\frac{2 * \text { Precision } * \Re \text { call }}{\text { Precision }+\mathfrak{R} \text { call }}$

464 For the 'uncertain' predictions, with more than one class attaining equal maximum score, it was 465 judged as more equitable to consider them as Negative results in all cases, since their predictive 466 value is minimal in practice (i.e. the true conformation may be one or none of those reported). 467 For those cases, if the true conformation of a CDR matches the major cluster in CDR/length, then 
that prediction counted as a False Negative for all further calculations - and as a True Negative in

469 the case of the true conformation not matching the major class.

\section{Post-evaluation DCP training and canonical templates' updating} experiment was performed where both the training set and the validation set were combined and subsequently used for DCP training and canonical templates' updating. The DCP parameters were retained from the previous validation step, meaning that parameters were not re-validated in this phase. Then, a final evaluation was performed on the test set. This stage was called 'Phase 2' and was analogous to a single cycle holdout experiment (Table 3). Phase-2 allowed an appreciation of the methods' performances in time, as more antibody structures become available.

\section{Publicly available prediction tool}

A GUI was developed with the Java Swing API for a computational tool that implements the prediction algorithms described for DCP and canonical templates ( ' $y C D R p$ '). The package (a jar file and a definitions folder) is available for downloading and use as a stand-alone desktop application at the "Humanisation bY Design" website, hosted by Birkbeck College, London at url: http://www.cryst.bbk.ac.uk/ ubcg07s. The GUI guides the user to manually structurally number their input Fv sequences and the tool's initial release applies Phase-1 DCP signatures and canonical templates ('definitions') for CDR conformational prediction.

\section{RESULTS}

\section{Selected Interaction Frames for testing}

Although during validation 3 IFs were assessed, in the following comparison of prediction results only the IF neighbourhood radius that gave the best predictive accuracy was considered. Table 4 shows the IFs that gave the best prediction results and their corresponding CDR neighbourhood radius. Positions at the end of the IF, marked as ' $n-x^{\text {' }}$, refer to CDR-H3 positions at a sequential distance $\mathrm{x}$ from the last residue $\mathrm{n}$ (H102). Since CDR-H3's length is 
hypervariable, it was found that this notation better reflects the topological equivalence of numbered positions.

Notations ' $\mathrm{E}$ ', ' $\mathrm{K}$ ' and ' $\mathrm{K}^{+}$', at the end of the $\mathrm{CDR}-\mathrm{H} 3$-base IF, refer to the $\beta$-hairpin type that is favoured at the CDR-H3 apex, depending on the formation of an Extended E (Extended

504 Negative $E^{\mathrm{N}}$ and Extended Positive $E^{\mathrm{P}}$ both resulting in the same $\beta$-hairpin ladder), single-bulged 505 Kinked $(\mathrm{K})$ or Kinked with double-bulged $\left(\mathrm{K}^{+}\right)$base. The hypothetical $\beta$-hairpin types $(\mathrm{A} / \mathrm{B} / \mathrm{C} / \mathrm{D})$ were derived from the definitions of the base type in Shirai, Kidera \& Nakamura (1999). The profiling of an IF fragment that contains a hypothetical $\beta$-hairpin type would give the following correspondence in English: "is the co-existence of specific residues at specific Fv positions with a hypothetical $\beta$-hairpin type in CDR-H3 distinct within a class and therefore a disjoint event between different classes?" These categorical IF positions were introduced experimentally to the CDR-H3 IF and proved beneficial in practice. It was thus demonstrated that IFs may also include categorical features (another categorical example would be the CDR length) in order to allow the consideration of more complex combinations, for instance between residues and structural

514 features.

\section{Summary results for all experiments}

Tables 5 and 6 show the accuracy of each method in each subset and experiment. Novel/non-previously clustered conformations observed in the new dataset are removed from the totals, in order to only assess performances on conformations that are predictable. Similarly, structures with a CDR length that contained less than 10 unique sequences in the clustered set were not considered. Canonical templates' results show a reduced total test population in CDRL3, because no templates were available for a length of 11-residues. Individual results are commented on later, per corresponding CDR.

All predictions for every CDR in the test sets, along with a measure of RMSD distance of the Query conformation from the closest cluster medoid, can be consulted in detailed tables in supplementary material: 'individual_CDR_predictions.xls'. Detailed tables with accuracy ratios per CDR/length, as well as extended statistics measuring the methods' performance in predicting

529 the major cluster in each CDR/length, are presented and commented on below (Tables 7-8 for 530 CDR-L1/L3, Tables 9-10 for CDR-H1/H2 and Tables 11/12 for CDR-H3-base). In order to allow 531 a direct comparison between methods, cumulative results (i.e. the entire new dataset) are 
532 considered in these detailed tables, but summaries of each method's performance per subset and

533 per experiment are also separately provided (Tables 5 and 6).

Predictions for CDR-L1

For the DCP method in CDR-L1 predictions, validated training parameters were a $6 \AA$ IF 538 and a combinatorial order up to triplets. The entire clustered dataset was used during training 539 (excluding outlying members in order to reduce the complexity of the predictable testing 540 structure). The method achieved an overall ratio of accurately predicted CDR-L1 of 92\% 541 (163/177), while the number of novel conformations in the test set, represented another 7\% 542 (12/177; Table 7). Of special note is the fact that in 12- and 13-residue CDR-L1 lengths, 543 classified as non-predictable or statistically uncertain ('type III') in North, Lehmann \& Dunbrack 544 (2011), the method predicted successfully $79 \%$ and $94 \%$ of the test CDRs respectively $(11 / 14$, $5451 / 14$ being a novel conformation, and 16/17, 1/17 being novel, respectively). This becomes more 546 striking when considering that $79 \%(11 / 14)$ and $88 \%(15 / 17)$ respectively of the test CDR sequences were new and not represented in the training set.

$548 \quad$ Predictions with canonical templates were overall accurate in $88 \%(155 / 177)$ of the test set (Table 7). This prediction score was mainly lowered by the existence of a number of uncertain predictions $(\sim 5 \%, 9 / 177)$, where more than one conformation achieved the same maximum canonical matching. As far as the prediction performance towards the major cluster in each length

552 is concerned (level-1 clusters: class-I; Nikoloudis, Pitts \& Saldanha, 2014, PeerJ preprint server, 553 DOI: 10.7287/peerj.preprints.291), DCP signatures proved superior to or as effective as canonical 554 templates in most measures, except for the precision in 12-residue CDR-L1 (0.88 vs. 1.0); and 555 also accuracy (0.95 vs. 1.00) and precision (0.95 vs. 1.00) in 13-residue CDR-L1 (Table 8). In 556 both lengths, this result is due to the fact that canonical templates output an uncertain prediction 557 in actual conformations other than the one corresponding to the major cluster in that length (i.e., 558 not a class '-I' tag), which technically counted as True Negatives in our assessment. These True 559 Negatives increased the respective accuracy and precision scores for the canonical model. In 560 contrast, canonical templates scored very low in recall in 12-residue CDR-L1 (0.44) vs. DCP 561 (0.88). Concerning the most voluminous cluster in CDR-L1, i.e. L1-11-I (160/434 or $\sim 37 \%$ of 562 total CDR-L1 clustered sequences; Nikoloudis, Pitts \& Saldanha, 2014, PeerJ preprint server, 563 DOI: 10.7287/peerj.preprints.291), both methods performed equally well with an F-measure of 5640.98 (Table 8), while 65\% (70/107) of the test CDRs were new sequences (Table 7). Thirty-three 
565 structures had a CDR-L1 length where only one cluster was available (7, 10 and 17 residues), or

566 less than 10 unique sequences were available during clustering (9 residues). Two structures had a 567 CDR-L1 with a new, non-clustered length (8 residues).

568 After discarding non-predictable CDRs (novel conformations, very low clustered 569 populations, or only one cluster per length), DCP signatures achieved an overall accuracy of $99 \%$ $570(163 / 165)$, as compared to $94 \%(155 / 165)$ for canonical templates (Table 2). Although both 571 methods performed very well, DCP signatures' performance proved slightly superior in all 572 individual assessments. However, post-evaluation DCP training and re-assessment (Phase-2) on 573 the test set, resulted in two wrong and one correct prediction switches, resulting in a roughly $1 \%$ 574 lowering of the final accuracy of the method (Table 5; comparison between "Initial" and 575 “Updated DCP signatures, Evaluation: test set”). Accordingly, post-evaluation updating of 576 canonical templates didn't have any effect on the predictions obtained for the new dataset $(96 \%$ 577 in both cases). Results suggest stability of both methods in view of the new structures, although 578 assessment with bigger test sets will be required in the future for a safer conclusion.

\section{Predictions for CDR-L2}

Nearly all 178 new structures with a Light chain had a CDR-L2 belonging to cluster L2-7I (175/178, 98\%). This was expected, since over 96\% (272/282) of the clustered CDRs had this conformation (Nikoloudis, Pitts \& Saldanha, 2014, PeerJ preprint server, DOI: 10.7287/peerj.preprints.291). Moreover, conformational differences between the three observed clusters are rather minimal (mostly peptide flips that additively result in inter-cluster medoid distances ranging between $1.1 \AA$ and $1.36 \AA$ ) and can be marginally characterised as variants of

588 the main L2-7-I conformational theme. For these reasons, the predictive methods were not applied in CDR-L2. Future work targeting, with greater precision, the variants within a cluster could reveal whether these conformational differences are predictable by sequence alone, or even

591 the result of experimental errors.

\section{Predictions for CDR-L3}

For the construction of CDR-L3 DCP signatures, a $4 \AA$ IF was used and for detection of disjoint signals, IF fragment combinations were examined up to triplets. The DCP method achieved an overall ratio of accurately predicted conformations of $85 \%$ (152/178), while $7 \%$ 
598 (13/178) of the test set were novel conformations (Table 7), of which 12/13 had new CDR 599 sequences (supplementary material: 'individual_CDR_predictions.xls'). The lowest accuracy was 600 observed in 10-residue CDR-L3; a length that, interestingly, seems hypervariable in 601 conformation. From the 27 unique sequences of CDR-L3/10-residues in the clustering dataset, 12 602 clusters were formed each containing between 1 and 4 unique CDR sequences, while 6 more 603 CDRs were labelled as outliers/singletons (Nikoloudis, Pitts \& Saldanha, 2014, PeerJ preprint 604 server, DOI: 10.7287/peerj.preprints.291). Nonetheless, since 7/15 test structures with CDR605 L3/10-residues were novel conformations (all with new CDR sequences; Table 7) it was 606 impractical to make any conclusions regarding predictive efficacy in this CDR length. The 607 biggest cluster in CDR-L3 is L3-9-I containing 328/480, or $\sim 68 \%$, of the clustered, non608 redundant structures, all CDR-L3 lengths considered (Nikoloudis, Pitts \& Saldanha, 2014, PeerJ 609 preprint server, DOI: 10.7287/peerj.preprints.291); a percentage that is interestingly verified in 610 the present new dataset (119/178, or $67 \%$ of the unique CDR-L3 sequences, Table 7$)$. For this 611 cluster, the DCP method achieved an accuracy of 0.93, while its F-measure was 0.96 (Table 8).

612 The canonical model achieved comparable overall prediction accuracy of $86 \%(131 / 153)$, 613 excluding predictions for CDR-L3/11-residues as no template was available (Table 7). For a 614 direct comparison, total accurate predictions for DCP signatures for 8-, 9- and 10-residue CDR615 L3 were 133/153 (87\%). Canonical model's score was lowered, for this method as well, mainly 616 by the presence of many novel conformations in CDR-L3/10-residues, and also a few uncertain 617 predictions obtained in CDR-L3/8- and 9-residues. Overall, both methods performed equally 618 well, with an only marginal superiority of the DCP method in CDR-L3/8- and 9-residues. 619 Canonical templates also achieved a slightly better score in accuracy (0.95 vs. 0.93) and precision 620 (0.96 vs. 0.93) of the major class-I in CDR-L3/9-residues (Table 8), which is again due to the fact 621 that uncertain predictions output for non-class-I conformations technically counted as True 622 Negatives. No predictions were made for a length of 5-residues as only one cluster was available, 623 and for 12- and 13-residues as less than 10 unique CDR sequences were available in the clustered 624 data (6 and 3, respectively; Nikoloudis, Pitts \& Saldanha, 2014, PeerJ preprint server, DOI: $62510.7287 /$ peerj.preprints.291).

626 Initial cumulative performances after removing non-predictable conformations were 627 comparable: 92\% (154/167) for DCP signatures and 91\% (131/144) for canonical templates 628 (Table 5), although DCP was applied in one additional length (11-residues). In all individual 629 assessments DCP performed equally or slightly better, while both methods took equal benefit 630 from post-evaluation training/updating (Phase-2), gaining roughly $2 \%$ in overall accuracy (Table 
631 5, comparison between "Initial" and "Updated DCP signatures/canonical templates, Evaluation:

632 test set").

633

634 Predictions for CDR-H1

635

636

637

For the construction of DCP signatures for the CDR-H1 prediction, training parameters were the following: an $8 \AA \mathrm{IF}$ and fragment combinations up to triplets. Clustered populations in 638 CDR-H1 lengths 13- and 15-residues represented 96\% (446/465) of the total non-outlying, non639 redundant CDR-H1 population (Nikoloudis, Pitts \& Saldanha, 2014, PeerJ preprint server, DOI: 64010.7287 /peerj.preprints.291) and are the only ones that formed more than one conformational 641 cluster. The DCP method achieved an overall ratio of correct predictions of $87 \%$ (182/210), while $6429 \%(18 / 201)$ were novel/non-clustered conformations (Table 9), most of which were observed in 643 13-residue CDR-H1. The method's performance was rather poor in 15-residue CDR-H1 (56\%, $6445 / 9$ accurate predictions); although the small number of test structures in this length doesn't allow 645 any concrete conclusion. It is notable that two out of three structures with H1-15-II 646 conformations (3TJE, 3THM, supplementary material: 'individual_CDR_predictions.xls') were not predicted correctly - all 3 represented by new CDR-H1 sequences. A possible reason for this could be the small training population for the 3 clusters in CDR-H1/15-residues (24/2/1 unique sequences, respectively; Nikoloudis, Pitts \& Saldanha, 2014, PeerJ preprint server, DOI: 10.7287/peerj.preprints.291). In CDR-H1/13-residues, 88\% (177/201) were accurately predicted, $4 \%(8 / 201)$ were false predictions, and 8\% (16/201) were novel conformations (Table 9).

Generated canonical templates for CDR-H1 displayed an increased degree of allowed 653 residues' overlap. This was expressed by an increased number of uncertain predictions 654 (maximum score by more than one template): $26 / 210$, or $12 \%$ (Table 9). The overall ratio of 655 accurate predictions was $74 \%$ (156/210), while false prediction represented approximately 5\% 656 (10/210) (Table 9). Comparing the performance of both methods in predicting the major clusters 657 in each CDR-H1 length, the DCP method was from marginally to significantly superior to the 658 canonical model in all measures (Table 10).

After removal of non-predictable conformations, DCP signatures achieved a cumulative 660 accuracy of $95 \%(182 / 192)$ as opposed to the canonical model with $81 \%(156 / 192$; Table 6$)$. The 661 performance of DCP signatures was accordingly superior in all individual assessments, while 662 post-evaluation training/updating benefited both methods by $\sim 1 \%-2 \%$ (Table 6 , comparison 663 between "Initial" and "Updated DCP signatures/canonical templates, Evaluation: test set"). 


\section{Predictions for CDR-H2}

Predictions for CDR-H2 conformation concerned three lengths, where there were more

668 than one cluster and more than 10 unique clustered CDR sequences: 9-, 10-, and 12-residue 669 CDR-H2. Length 10-residues was of additional interest as it represented the only case, all CDRs considered, that featured both a considerable total population (350 unique clustered sequences) and two well-populated clusters with an approximate 1:2.5 ratio in non-redundant members. For 672 the construction of DCP signatures, a $6 \AA$ IF was employed and fragments up to quadruplets were compared. The method achieved accurate predictions in $89 \%$ of the test CDRs $(193 / 217)$, made an uncertain prediction in 6 cases $(\sim 3 \%)$, and a false prediction in 14 cases $(\sim 6 \%)$, while 4 more CDRs were novel conformations ( $2 \%$, Table 9$)$. For CDR-H2 lengths 9 - and 10-residues alone, North, Lehmann \& Dunbrack (2011) reported a theoretical percentage of correct predictions of $80 \%$, consulting extensively the identity of the residue at position $\mathrm{H} 71$, and of $78 \%$, using hidden Markov models.

Canonical templates for CDR-H2 displayed a very pronounced degree of overlap between allowed residues, which was even more severe than was observed in CDR-H1. This caused the percentage of uncertain predictions to rise to $34 \%$ (74/217), while the false predictions were $7 \%$ (16/217). The overall accuracy was therefore only 57\% (123/217, Table 9). Canonical performance in predicting the major cluster in each length suffered accordingly, although not as dramatically as the global accuracy would suggest (F-measure: 0.79 for CDR-H2/9-residues, 0.74 for CDR-H2/10-residues, Table 10).

In the case of CDR-H2 then, the advantage of comparing combinations of residues (DCP) was observed in a more prominent manner. Therefore, observations here support the initial hypothesis of the degree of sequence-to-structure residue synergistic complexity and the nonlinear determination of conformation by local and neighbouring residue preferences. However, post-evaluation DCP training resulted in the severe reduction of signals in the signatures of clusters H2-10-I and H2-10-II. This led to an increased rate of uncertain predictions during reevaluation with the updated signatures, which was reflected by a $6 \%$ loss in accurate predictions

693 (Table 6, comparison between "Initial" and "Updated DCP signatures, Evaluation: test set"), 694 although only less than 1\% loss when comparing the respective cumulative results (Table 6). Re695 updating of canonical templates, on the other hand, resulted expectedly in a slight decrease in accuracy $(\sim 1 \%)$, as updating could only accentuate the existing template overlap effect; although 
697 cumulatively, re-updating increased the ratio of accurate predictions by 2\% (Table 6). Normally,

698 this behaviour of DCP would suggest the need for re-parameterisation, using e.g. an increased 699 order of combinations (e.g. quintets) in order to preserve predictive performance in time. 700 Nonetheless, since the difference in global performance of the two methods is already so 701 dramatically in favour of the DCP method (Tables 6 and 9), it was judged preferable to 702 demonstrate rather than attenuate this effect, as a useful piece of critical assessment for this new 703 method that will allow improved future implementations.

\section{Predictions for CDR-H3-base conformation}

The pronounced sequence, length and conformational hypervariability in CDR-H3 was verified during the clustering (Nikoloudis, Pitts \& Saldanha, 2014, PeerJ preprint server, DOI: 10.7287/peerj.preprints.291) and in this landscape of variability it was completely impractical to apply predictive DCP on the complete CDR-H3 conformation, at least not in the form of the current implementation of this new method; a remark also arising from the earliest conception of the canonical model. Nonetheless, as a major advance in the prediction of CDR-H3 conformation from sequence concerns the formulation of sequence rules for the CDR-H3-base, DCP was applied on three CDR-H3-base categories: Kinked $(\mathrm{K})$, Extended negative $\left(\mathrm{E}^{\mathrm{N}}\right)$ and Extended positive $\left(E^{P}\right)$. Prediction of the double-bulged Kinked base $\left(K^{+}\right)$, was not attempted on this occasion for simplicity.

For DCP signatures, a $4 \AA$ IF was used and compared fragments up to quintets were compared. The DCP method made 195/216 correct predictions (90\%) for the CDR-H3-base conformation. Comparatively, application of sequence rules resulted in 183/216 (85\%) accurate predictions, for both the 1999 (Shirai, Kidera \& Nakamura, 1999) and 2007 (Kuroda et al., 2008) sets of rules respectively (Table 11). More specifically, the updated set of rules resulted in 11 correctly switched predictions $(\sim 5 \%)$ and 13 falsely switched predictions $(\sim 6 \%$, two switches were from a wrong prediction to another wrong prediction, the rest were from correct to wrong); 21 incorrect predictions made by the original set were retained in the 2007 set $(\sim 10 \%$; supplementary material: 'individual_CDR_predictions.xls').

The methods' performances were evaluated separately in predicting the Kinked base,

728 which represents the most frequent base conformation (roughly 7:2:1 ratio between $\mathrm{K}-\mathrm{E}^{\mathrm{N}}-\mathrm{E}^{\mathrm{P}}$ 729 conformations in all datasets combined). The updated rule set presented an almost identical 
730 performance, over all measures, to the original set (Table 12). It therefore cannot be verified that

731 the updating of sequence rules on the basis of new structures is beneficial; it can be argued that a

732 point may appear where the rules' predictive performance may no longer warrant their increasing

733 complexity. In comparison, predictions with DCP signatures brought a slight improvement over

734 both sets of sequence rules, all measures considered (accuracy 0.90 vs. 0.85, F-measure 0.95 vs.

735 0.92, Table 12). Although this improvement is still marginal, it confirms the new method's

736 consistency in out-performing, or performing at least as well as, the existing methods in all CDRs

737 including CDR-H3.

738

739 Discussion

The historical approach used for CDR prediction from sequence alone relies on canonical templates or in simpler cases the existence of a single conformational cluster for a given CDR length. For the hypervariable CDR-H3, where sequences, lengths and conformations show great diversity, sequence rules were formulated in order to allow the prediction of only the base of the loop. While the canonical model has been, and still is, effective in predicting a number of CDR conformations, its strength is inevitably weakened as more antibody structure become available. As the construction of canonical templates consists of identifying structurally-determining residues at specific positions that are exclusive to each canonical class, the proliferation of sequences in CDR clusters gradually creates overlapping, or rogue, templates (not to be confused with the multi-conformation, full-rogue CDRs in this work). This was first observed by Martin \& Thornton (1996) and was acknowledged by North, Lehmann \& Dunbrack (2011) where canonical templates discreetly gave way to statistical consensus sequences.

A typical problem with canonical templates, and by extension with statistical consensus sequences, is that they require the presence of previously observed residues in specific positions, without the consideration that certain overlapping combinations of residues may render the targeted CDRs unpredictable. While this could be statistically acceptable in the past as far as positive predictability was concerned, the great increase of CDRs in the PDB results in conformational clusters with highly overlapping canonical templates or consensus sequences; for example in CDR-H2/10-residues, all 14 canonical positions contain at least one overlapping residue between one or more other templates of the same length (supplementary material: 'all_Canonical_Templates.doc'). Additionally, another fact that becomes prominent with richer 
762 datasets is that many CDR conformations do not depend solely on their own sequence but receive 763 structurally-determining influence from the antibody's framework (Tramontano, Chothia \& Lesk, 764 1990; Martin \& Thornton, 1996; Morea et al., 1997; North, Lehmann \& Dunbrack, 2011). These 765 problems can sometimes be dealt with by application of Hidden Markov Model (HMM) analysis. 766 However this requires a considerable number of cluster members for the model to remain 767 reliable, and to some extent removes the simplicity that made the canonical model attractive in 768 predicting the conformations of antibody CDRs. Nonetheless, sequence logos were constructed 769 for the training clusters using Berkeley’s WebLogo facility (http://weblogo.berkeley.edu/logo.cgi) 770 (Crooks et al., 2004) and are provided as supplementary material ('all_sequence_logos.pdf').

As the present predictive methods essentially predict a class of similar conformations and not the actual CDR coordinates, their utility as far as modelling the loop is concerned is to be complemented by the cluster and sequence characteristics tied to the predicted class. Since the present work was based on the clustering analysis presented in Nikoloudis, Pitts \& Saldanha (2014, PeerJ preprint server, DOI: 10.7287/peerj.preprints.291), after a positive prediction, the members/structures corresponding to the cluster's (level-1) medoid and extremities should be extracted in order to get a quick appreciation of the extents of possible (known) conformational variability in the Query structure. A multiple alignment within that cluster's sequences would result in the members most similar to the Query sequence, one of which would form a preliminary template. Depending on the selection of the most appropriate template and any other CDRs to be modelled, a possible conformational shift towards the core or the extremities of the cluster's level-1 conformational theme would be inferred. Furthermore, consultation of other sequences of members belonging to the same level-2 or even level-3 cluster as the template, would reveal if specific sequence differences observed in the Query sequence are allowed within that specific variant. Finally, sequence features observed at the daughter-level could potentially also guide the modelling of the subtle conformational characteristics of that sub-cluster (i.e. at levels 2/3) with regard to the main/parent conformational theme that was predicted (i.e. at level788 1).

Based on the present prediction results, a conclusion that can be drawn regarding the canonical model is that it still presents an acceptable predictive capability, at least in most Light chain CDR lengths. Overall, accurate predictions by canonical templates were 565/757 (74.6\%)

792 in CDR-L1, -L3, -H1 and -H2, with 47/757 ( 6\%) being novel, non-predictable conformations

793 (sum of results in Tables 7 and 9); after removal of non-predictable conformations, total 
794 cumulative accurate predictions were then $565 / 710$ or $79.6 \%$ (sum of results in Tables 5 and 6).

795 Its performance in Heavy chain CDRs though, where the overlap of canonical templates resulted 796 in important accuracy loss (Tables 6 and 9), could suggest that the efficacy of the canonical 797 model may be bound to decrease over time as more structures become available. One possible 798 solution for retaining the practicality of the canonical model could be a k-fold cross-validation 799 analysis of a dataset in order to obtain the canonical templates that best predict the available 800 conformations; and then keep those templates locked until the assessed performance of the model 801 begins to decline again in the future. Alternatively, the re-sampling of established canonical 802 positions in each CDR length could also potentially result in better performance, i.e. a cross803 validation analysis with reduced sets of canonical positions. Such a process is expected to 804 virtually remove several heavily overlapping positions and allow better template specificity.

805 Following a much more supervised approach, sequence rules used for prediction of CDR$806 \mathrm{H} 3$ features still demonstrate a satisfactory predictive potential as confirmed by the blind-testing 807 sessions. With an overall accuracy of $85 \%$ during testing over the two sets of rules, it can be 808 supported that the sequence basis for the CDR-H3-base conformation is essentially acquired. On 809 the other hand the addition of 8 new rules or rule-adaptations in the updated set, on top of the 810 original 4, didn't procure an improvement in accuracy. It can be argued that the test set of 216 811 sequences was relatively small for safe conclusions, compared to the 311 sequences used during 812 the formulation of the updated rules. However, these additional rules were created for the correct 813 identification of only 47/311 (15\%) of CDR-H3-bases that were misclassified by the original set 814 of rules (Kuroda et al., 2008). Interestingly, exactly the same percentage of misclassified bases 815 (15\%; Table 11) was again also displayed by the updated rules during the testing session, 816 suggesting a possible attained limit in the efficacy of the sequence rules. Moreover, false switches 817 from the original set's prediction were not avoided (13 cases), while the correct prediction 818 switches were fewer than the number of false predictions retained from the original set (11 and 819 21, respectively in supplementary material: 'individual_CDR_predictions.xls'). Therefore, the 820 test set was generally representative of the predictive challenge a researcher may encounter and, 821 as previously mentioned, that sequence rules could already have reached a point where their 822 further specialisation towards improvement of accuracy has become impractical, ineffective, or 823 both.

824 The newly proposed predictive method (DCP) achieved an overall score of correct 825 predictions in all examined CDRs of 88.7\% (885/998), while approximately 5\% (47/998) of the 826 test CDRs represented novel, unpredictable conformations (sum of results in Tables 7, 9 and 11); 
827 after removal of non-predictable conformations, total cumulative accurate predictions were then

$828885 / 951$ or $93.1 \%$ (sum of results in Tables 5 and 6). The improvement over the canonical model 829 or sequence rules was consistent in all CDRs, ranging from 1\% in CDR-L3 to 33\% in CDR-H2 830 (average 10.8\%, median 6\%) cumulatively over the entire new dataset (comparison between 831 cumulative results in Tables 5 and 6), and ranging from $2 \%$ to $30 \%$ (average 9.7\%, median 5.5\%) 832 over the test subset only (initial evaluation of test set, Tables 5 and 6). This improvement was 833 verified during the evaluation of prediction performance for the most populated, and thus 834 statistically most important, cluster in each predictable length. Over 60 total measures (15 835 common categories, 4 statistical evaluations per category), the DCP method's score ranged from 836 equal to significantly better in all but 6 cases, in which canonical templates performed marginally 837 better mainly due to technicalities of the assessment that were discussed previously (Tables 8,10 838 and 12). With all but two F-measure scores (L3/10-residues, H1/15-residues) being better than 8390.88 (average 0.90 , median 0.96 ), confidence for accepting or rejecting the adoption of the major 840 conformation in length by the unknown CDR can be relatively high.

841 This performance was deemed encouraging, considering the method's novel and 842 embryonic nature. It can therefore also be argued that the threshold-free approach of the initial 843 clustering was advantageous for prediction as it created richer clusters by including more 844 sequence examples and possible variants of a conformational theme. These variants could have 845 diminished the predictive efficiency of the assessed methods, if considered as separate clusters in 846 the first place. Indeed, unless these variants were later detected as multi-conformation full-rogues 847 which would lead to their predictive merging, their separation from the main conformational 848 theme would produce poorer training/updating results due to considerably fewer examples per 849 profiled cluster. In any case, it would also be interesting to apply DCP at levels -2 and -3 of the 850 nested initial classification, in order to explore the potential of prediction of the more subtle 851 variants, which would be of increased importance to antibody engineering, if successful. 852 Moreover, future dedicated work on DCP signatures may bring further improvements in the 853 overall predictive potential by proposing more elegant implementations than the basic approach 854 employed in this work.

855 Clusters that contained members with the exact same Fv sequences were merged for 856 training/updating and prediction. Hence, DCP signature or canonical template matching of a 857 combined predictable conformation reported all the affected conformations at the same time. In 858 these cases there was inevitably a loss of specificity towards the prediction of each separate 859 conformation. However in practice, in 295/301 ( 98\%) related cases of accurate prediction by 
860 DCP signatures of a combination of clusters, the true conformation was always that of the major

861 class of the set. This could suggest that those smaller clusters that contain multi-conformation

862 full-rogue CDRs are more valuable for merely being part of the known conformational repertoire

863 of that CDR and for becoming the object of sequence-to-structure and/or CDR induced-fit

864 studies, rather than representing important predictable conformations.

865 Alternatives to the above approach for successful training would be to exclude the 866 sequences of all involved members from their respective cluster sets, or to exclude the sequence 867 sets of the smaller cluster altogether as not important for prediction; both scenarios hiding 868 potential training inconveniences. Therefore, the predictive cluster merging preserves the 869 availability of sequence information, did not practically reduce prediction sensitivity and 870 presented no obvious bias toward one of the two predictive methods that are compared for each 871 CDR. On the contrary, most merges may be pointing to closely related conformations whose 872 divergence is due to external factors, in which case it makes more sense to consider them in a 873 combined fashion.

874 Perhaps the biggest future challenge for the DCP method would be to detect the presence 875 of a novel class - not the novel conformation itself - but merely the potential to avoid a false 876 positive identification. This is an inconvenience shared by all sequence-based methods, since 877 they always attribute a class to an unknown structure. The avoidance of false positives (all classes 878 considered) could be achieved in time as signatures become more specific, in which case a 879 positive identification would require a ratio score better than a defined threshold (e.g. no positive 880 prediction below $\mathrm{R}_{\mathrm{DCP} \text { signature }}<0.5$, Equation 1). Alternatively, this could be achieved with the 881 definition of a negative class. The training protocol of the DCP method may indeed allow for 882 such a process, precisely because it is searching for differences between the compared IF 883 sequence sets instead of similarities. An exploratory approach could be the selective mixing of 884 different classes divided between Query and Target sets, in order to represent a non-existent 885 conformation or combinatorial chimera, for profiling of disjoint combinations. Signatures 886 obtained from such training should then be tested for positively attracting unknown 887 conformations, without interfering with known classes.

888 The biggest culprit during DCP training was undoubtedly its execution complexity which 889 scales in factorial time. In practice with a short $4 \AA \mathrm{IF}$, single-threaded execution time was 890 acceptable for DCP with IF sequence fragments up to quintets, or even sextets (i.e. up to 2-3 891 minutes per CDR). However with longer (up to $8 \AA$ ) IFs, execution time becomes very quickly 892 prohibitive, with quartets' training requiring sometimes close to 50 minutes per CDR on the 
893 available computational setup (2.67MHz Intel i5 quad-Core processor). As was revealed by the 894 test results, supervised exploration of a number of selected [IF length]/[fragment order] 895 combinations of training sets proved sufficient in order to reach and surpass the performance of 896 the other established methods. However for optimisation of DCP signatures, a k-fold cross897 validation of the signature signals may be required, which will be the focus of a future study. Of 898 course, it cannot be ruled out that future dedicated studies may also propose a more efficient 899 training procedure, e.g. by defining shorter IFs based on a criterion other than the structural 900 neighbourhood of a CDR. Also, another way for producing more accurate and specific DCP 901 signatures could be in the statistical validation of the disjoint combinations/events. Toward this 902 end, a probabilistic closed-form equation for selecting only statistically significant signature 903 signals is proposed as an appendix in supplementary material:

904 'appendix_Statistical_Validation.doc'. As a final suggestion, the representation of IF sequence 905 sets using reduced non-overlapping amino acid alphabets is another intriguing possibility to be 906 explored for an improved implementation of the DCP method.

907 It should be noted that both DCP signatures and canonical templates are by design able to 908 achieve a maximum score with the totality of a training set, but with DCP an uncertain retro909 prediction is not possible. After post-evaluation training/updating on both the clustering and the 910 validation sets, re-evaluation of the validation set showed superior aptitude of DCP signatures in 911 retro-predicting the set they were trained upon (100\% correct predictions, Tables 5 and 6). This 912 behaviour was expected as DCP signatures capture all the combinatorial differences between one 913 class and all the others. Additionally, the IF sequence of any Query structure gets included into 914 the Target IF sequence sets of all clusters except for the one that corresponds to the Query 915 structure. Hence, no disjointness can be observed between the Query IF sequence fragments and 916 any non-corresponding cluster, so uncertain predictions are essentially avoided. In contrast, 917 canonical templates display a more linear ensemble of intra-class similarities that become 918 overlapping, which penalises the predictive accuracy of the model. This means that, at least in 919 theory, updating of DCP signatures by adding new sequences to the training set should produce 920 more stable and accurate predictive models. Thus, provided that an optimised set of training 921 parameters (IF radius and combinatorial order) is acquired, disjoint signals should become 922 naturally filtered and signatures should be increasingly specific to each class, as more structures 923 and their sequences bring additional examples of clustered conformational themes. Analysis of 924 the individual signals within these increasingly specific signatures could then potentially assist in 
925 discovering important interactions that contribute to CDR conformation; a feature that is not

926 easily accessible in other analytical methods such as HMM or neural networks.

927 As a random example of a possibly interesting combination of residues from the obtained

928 signatures of cluster L1-11-II, a prominent detected disjoint couplet that is retained in phase-2

929 signatures and also potentially validated as significant by absolute probabilistic significance

$930(\mathrm{p}=0.0012)$ was L5/L66. Beside the fact that both positions of that combination are not included

931 in any CDR-L1 canonical template, it is interesting to note that positions L5 and L66 are located

932 at laterally opposite locations with regard to the L1 loop, with a $\mathrm{C} \alpha-\mathrm{C} \alpha$ distance usually around

$93312 \AA$ and therefore make no contact whatsoever with each other. Keeping all reservations

934 regarding the interpretability of this observation, due to the lack of a dedicated related study on

935 this occasion, this combination appears as a showcase of a possible chained synergy between

936 residues as mentioned earlier. Indeed, as the addition of any Fv position to an already disjoint

937 combination of positions results in a new disjoint combination by definition, then it could be

938 possible that in this case the method captured a chained effect starting at position L5 and

939 involving other or all topological positions between L5 and L66, located on the other side of the

940 L1 loop. Also interestingly, while position L66 is relatively conserved, hosting a Gly in most light

941 Fv sequences, this is apparently not the case in members of the L1-11-II cluster. Instead, the

942 couplet IF fragment sequences L5/L66 were detected to be always different between L1-11-II and

943 all the other clusters in that length. However, only a dedicated study would safely lend itself to

944 such an interpretation, in which case the actual observed combinations of residues of the disjoint

945 signals could play a role in the modelling of the respective cluster's loop conformation. Finally,

946 important interactions between residues that influence the VH/VL interface orientation can also

947 be potentially captured during DCP, since the defined Interaction Frames involve several residues

948 from both chains.

949 Important advancements are being made in other methods for the prediction of CDR 950 conformation or loop conformation in general. These include general ab initio modelling 951 techniques (e.g. Loopbuilder, Soto et al., 2008), fragment assembly techniques (e.g. 952 RosettaAntibody, Sivasubramanian et al., 2009; Weitzner et al., 2014), or database search 953 techniques (e.g. FREAD, Choi \& Deane, 2011). While the accuracy of these methods is typically 954 measured in average RMSD from the tested crystal structures, respective publications usually do 955 not mention the ratio of wrongly predicted conformations based on an acceptable RMSD 956 threshold, as was the case in this work. To allow future comparisons with such methods, it is 957 worth reporting that this new classification-based prediction method (DCP) presented an average 
958 RMSD (and median in $\AA$ ) after C $\alpha$-backbone superposition of CDR residues to the medoids of

959 the correctly identified class of 0.36(0.30)-0.40(0.33)-0.54(0.41)-0.36(0.32) for CDR-L1-L3-H1-

$960 \mathrm{H} 2$, respectively (figures calculated from "RMSD distance of observed conformation to cluster

961 medoid”, supplementary material: 'individual_CDR_predictions.xls'). For CDR-L1, -L3, -H1 and

962 -H2 with DCP signatures, only 30/692 ( 4\%) of correctly identified conformations had an RMSD

963 from the medoid greater than $1 \AA$ (1.03 $-1.58 \AA$, average $1,21 \AA$, median $1.19 \AA)$. A direct

964 comparison between the present implementations of the DCP/canonical templates' methods and

965 the aforementioned CDR modelling tools was not performed, but can be pursued in the future 966 using the GUI tool mentioned above.

968 Conclusion

A new predictive model was developed for CDR conformation, its training workflow was designed and a first application was demonstrated on a new test set of structures. Prediction performance was shown to be superior to previous sequence-based methods over all CDRs. The method permits increased parameterisation and presents implementation flexibility. These characteristics allow a considerable margin for performance improvement in future work, and also suggest the possibility that it can be exploited in other fields of biological research. To the best of our knowledge, there existed no similar method with the particular features of DCP at the time of development, i.e. the search for common differences represented by disjoint, mixed sequence combinations between sets of classified sequences, or classified instances in general. Therefore it can be claimed that the method is novel, original and adaptable. It proved impractical to fully verify whether other methods with similar characteristics or features were not developed in research areas other than the

982 biological arena, and therefore this possibility cannot be completely ruled out, e.g. in 983 document-related areas that perform intensive combinatorial operations such as 984 cryptography/decryption. Should this be the case, then only the claim of the method's novelty regarding the specific application to antibody CDR conformation was demonstrated

986 in this study. In any case and in conclusion, although the development of alternative prediction 987 methods is important, especially ones with an ab initio or fragment-based approach for predicting 988 novel conformations, it is suggested that the strictly sequence-based methods examined here fully 
retain their innate advantages in prediction time, input simplicity and conformational precision

990 upon positive identification.

991

992

993 REFERENCES

994

995

996

997

998

999

1000

1001

1002

1003

1004

1005

1006

1007

1008

1009

1010

1011

1012

1013

1014

1015

1016

1017

1018

1019

1020

Al-Lazikani, B., Lesk, A.M. and Chothia, C. (1997). Standard conformations for the canonical structures of immunoglobulins. J. Mol. Biol., 273, 927-948.

Alzari, P.M., Spinelli, S., Mariuzza, R.A., Boulot, G., Poljak, R.J., Jarvis, J.M. and Milstein, C. (1990). Three-dimensional structure determination of an anti-2-phenyloxazolone antibody: the role of somatic mutation and heavy/light chain pairing in the maturation of an immune response. EMBO. J., 9, 3807-3814.

Barré, S., Greenberg, A.S., Flajnik, M.F. and Chothia, C. (1994). Structural conservation of hypervariable regions in immunoglobulins evolution. Struct. Biol., 1, 915-920.

Berman, H.M., Westbrook, J., Feng, Z., Gilliland, G., Bhat, T.N., Weissig, H., Shindyalov, I.N. and Bourne, P.E. (2000) The Protein Data Bank. Nucleic Acids Research, 28, 235-242.

Choi, Y. and Deane, C.M. (2011). Predicting antibody complementarity determining region structures without classification. Mol. BioSyst., 7, 3327-3334.

Chothia, C., Lesk, A.M., Levitt, M., Amit, A.G., Mariuzza, R.A., Phillips, S.E.V. and Poljak, R.J. (1986). The predicted structure of immunoglobulin D1.3 and its comparison with the crystal structure. Science, 233, 755-758.

Chothia, C. and Lesk, A.M. (1987). Canonical structures for the hypervariable regions of immunoglobulins. J. Mol. Biol., 196, 901-917.

Chothia, C., Lesk, A.M., Tramontano, A., Levitt, M., Smith-Gill, S.J., Air, G., Sheriff, S., Padlan, E.A., Davies, D., Tulip, W.R., Colman, P.M., Spinelli, S., Alzari, P.M. and Poljak, R.J. (1989). Conformations of immunoglobulin hypervariable regions. Nature, 342, 877-883.

Chothia, C., Lesk, A.M., Gherardi, E., Tomlinson, I.M., Walter, G., Marks, J.D., Llewelyn, M.B. and Winter, G. (1992). Structural repertoire of the human $\mathrm{V}_{\mathrm{H}}$ segments. J. Mol. Biol., 227, 799-817.

Crooks, G.E., Hon, G., Chandonia, J.M. and Brenner, S.E. (2004). WebLogo: A sequence logo generator. Genome Research, 14, 1188-1190. 
1021 de la Paz, P., Sutton, B.J., Darsley, M.J. and Rees, A.R. (1986). Modelling of the combining sites

1022 of three anti-lysozyme monoclonal antibodies and of the complex between one of the 1023 antibodies and its epitope. EMBO J., 5, 415-425.

1024 Furukawa, K., Shirai, H., Azuma, T. and Nakamura, H. (2001). A role of the third 1025 complementarity-determining region in the affinity maturation of an antibody. J. Biol. Chem., $1026 \quad 276,27622-27628$.

1027 Guarne, A., Bravo, J., Calvo, J., Lozano, F., Vives, J. and Fita, I. (1996). Conformation of the 1028 hypervariable region L3 without the key proline residue. Protein Sci., 5, 1931-1933.

1029 Guex, N. and Peitsch, M.C. (1997). SWISS-MODEL and the Swiss-PdbViewer: An environment 1030 for comparative protein modelling. Electrophoresis 18, 2714-2723.

1031 Kabat, E.A., Wu, T.T. and Bilofsky, H. (1977). Unusual distributions of amino acids in 1032 complementarity-determining (hypervariable) segments of heavy and light chains of immunoglobulins and their possible roles in specificity of antibody-combining sites. $J$. Mol.

1035 Kuroda, D., Shirai, H., Kobori, M. and Nakamura, H. (2008). Structural classification of CDR1036 H3 revisited: A lesson in antibody modelling. Proteins: Struct. Funct. Bioinf., 73, 608-620.

Martin, A.C. and Thornton, J.M. (1996). Structural families in loops of homologous proteins: 1038 automatic classification, modeling and application to antibodies. J. Mol. Biol., 263, 800-815.

1039 Morea, V., Tramontano, A., Rustici, M., Chothia, C. and Lesk, A.M. (1997). Antibody structure, 1040 prediction and redesign. Bioph. Chem., 68, 9-16.

1041 Morea, V., Lesk, A.M. and Tramontano, A. (2000). Antibody modeling: implications for 1042 engineering and design. Methods, 20, 267-279.

1043 North, B., Lehmann, A. and Dunbrack R.L. Jr. (2011). A New Clustering of Antibody CDR Loop 1044 Conformations. J. Mol. Biol., 406, 228-256.

1045 Shirai, H., Kidera, A. and Nakamura, H. (1996). Structural classification of CDR-H3 in 1046 antibodies. FEBS Letters, 399, 1-8.

1047 Shirai, H., Kidera, A. and Nakamura, H. (1999). H3-rules: identification of CDR-H3 structures in antibodies. FEBS Letters, 455, 188-197.

1049 Sivasubramanian, A., Sircar, A., Chaudhury, S. and Gray, J.J. (2009). Toward high-resolution 1050 homology modelling of antibody $\mathrm{F}_{\mathrm{v}}$ regions and application to antibody-antigen docking. Proteins, 74, 497-514. 
1052 Soto, C.S., Fasnacht, M., Zhu, J., Forrest, L. and Honig, B. (2008). Loop modelling: Sampling, 1053 filtering, and scoring. Proteins, 70, 834-843.

1054 Tramontano, A., Chothia, C. and Lesk, A.M. (1990). Framework residue 71 is a major 1055 determinant of the position and conformation of the second hypervariable region in the $\mathrm{V}_{\mathrm{H}}$ 1056 domains of immunoglobulins. J. Mol. Biol., 215, 175-182.

1057 Tomlinson, I.M., Cox, J.P.L., Gherardi, E., Lesk, A.M. and Chothia, C. (1995). The structural 1058 repertoire of the human $\mathrm{V}_{\kappa}$ domain. EMBO J., 14, 4628-4638.

1059 Vargas-Madrazo, E. and Paz-García E. (2002). Modifications to canonical structure sequence 1060 patterns: analysis for L1 and L3. Proteins: Struct. Funct. Genet., 47, 250-254.

1061 Weitzner, B.D., Kuroda, D., Marze, N., Xu, J. And Gray, J.J. (2014). Blind prediction 1062 performance of RosettaAntibody 3.0: Grafting, relaxation, kinematic loop modelling, and full 1063 CDR optimization. Proteins: Struct. Funct. Genet., doi: 10.1002/prot.24534. (Epub ahead of 1064 print).

1065 Wu, T.T. and Kabat, E.A. (1970). An analysis of the sequences of the variable regions of Bence1066 Jones proteins and myeloma light chains and their implications for antibody complementarity. 1067 J. Exp. Med., 132, 211-250.

1068

1069

1070

1071

1072

1073

1074

1075

1076

1077

1078

1079

1080

1081

1082

1083

1084 
1085

1086

1087

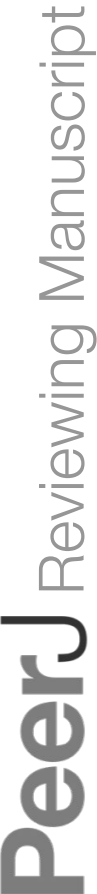

PeerJ reviewing PDF | (v2014:03:1771:1:1:NEW 26 May 2014) 


\section{Figure 1}

Preparatory steps for DCP.

Preparatory steps for DCP. Here, an Interaction Frame (IF) is selected for CDR-L1 and the corresponding IF sequences are synthesised for each one of the four clusters of the given length. For computational reasons the same IF is defined for all lengths of any given CDR (here, CDR-L1 for illustration purposes). Therefore, observed gaps in IF sequences correspond to insertions populated in longer lengths than the one shown in the illustrated example - gaps are filled accordingly in those lengths' IF sequences. Spare gaps, on the other hand, may correspond to IF positions pointing to unpopulated insertions from other CDRs or deletions in the Fv sequence. Also, gaps are present if there is no Light or Heavy chain in that particular structure. Positions at the end of the IF, marked as ' $n-x^{\prime}$ ', refer to CDR$H 3$ positions at a sequential distance $x$ from the last residue $n(H 102$; see text).

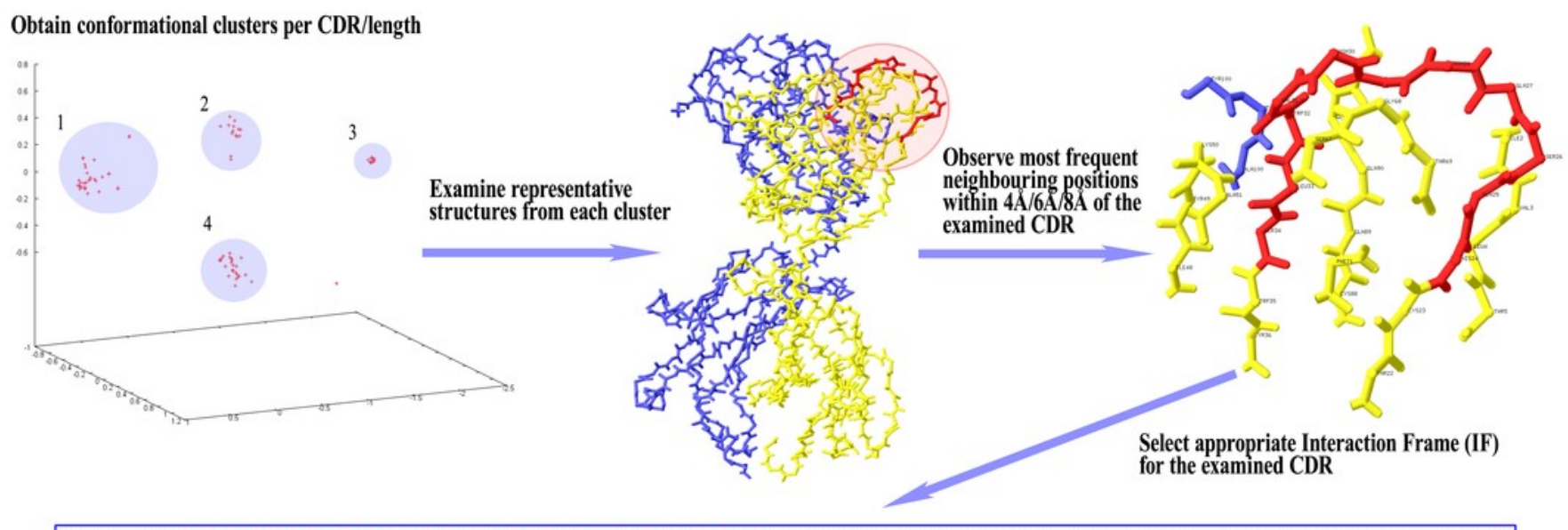

L2 L3 L4 L5 L22 L24 L25 L26 L27 L28 L29 L30 L30a L30b L30c L30d L30e L30f L31 L32 L33 L34 L36 L46 L48 L49 L50 L51 L52 L66 L67 L68 L69 L70 L71 L87 L89 L90 L91 L92 L93 L94 L95 H96 n-4 n-3 Synthesise corresponding IF sequences from all cluster members

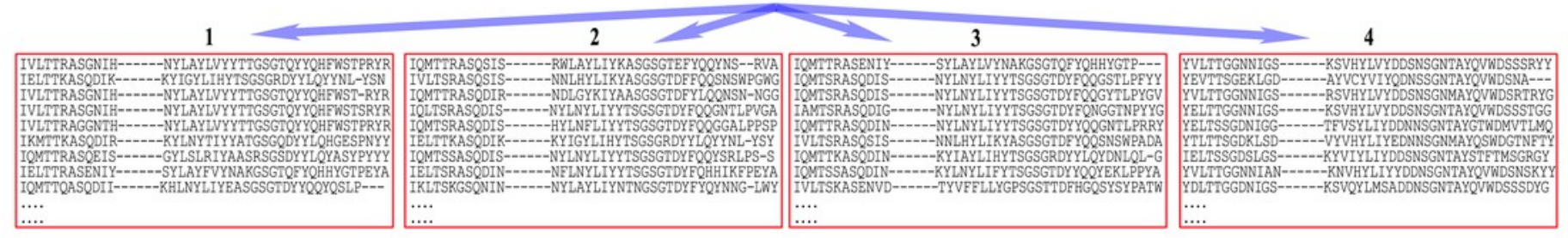




\section{Figure 2}

\section{The training procedure using Disjoint Combinations Profiling.}

The training procedure using Disjoint Combinations Profiling: definition of Query and Target

IF sequence sets, extraction of all available IF fragment sequences and comparison between corresponding sets of fragments for disjointness, leading to signature signals.

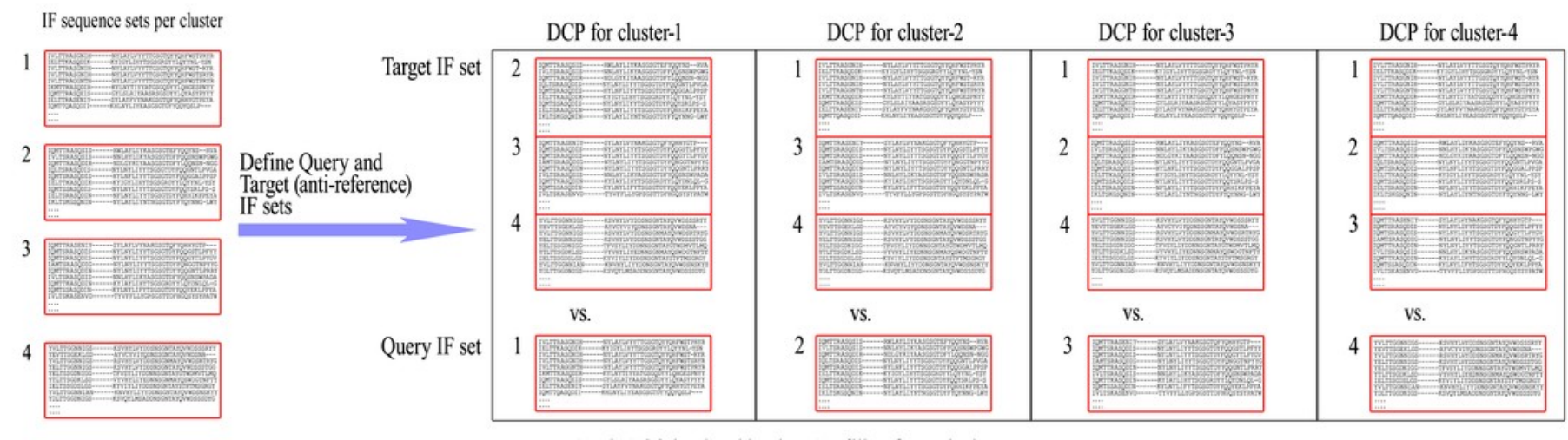

Begin Disjoint Combinations Profiling for each cluster

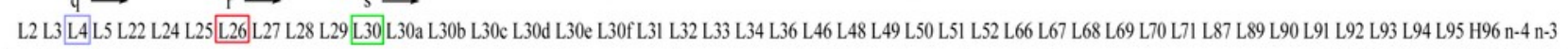
Construct all possible couplets/triplets/etc. sequence combinations If arrays are disjoint (no common fragments) from all IF sequence sets

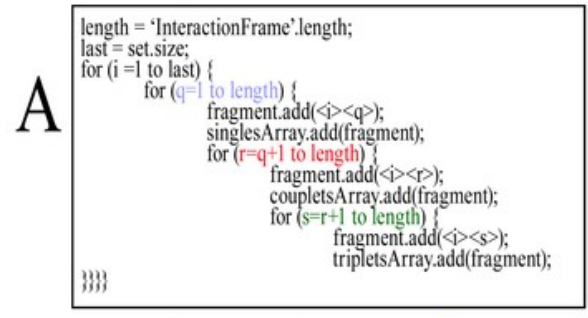

$\Rightarrow A D D$ fragment positions to cluster signature

Obtain complete DCP signature

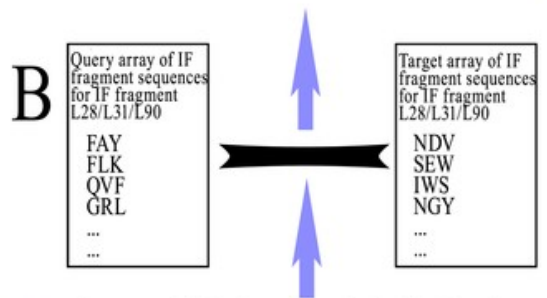
Compare all singletons/couplets/triplets/etc. Query and Target arrays consisting of signature signals

Repeat for Query and Target sets 


\section{Figure 3}

Representation of the workflow for CDR conformation prediction by DCP signatures.

New Fv sequences are referred to as "Query" sequences, as they become the profiled object, and therefore IF fragment sequences from the new Fv sequences become 'Query IF fragment sequences' for the purposes of prediction. 
1 Numbered query Fv sequence, unknown CDR conformations

1 heAVY CHAIN

DVQL-QESGPGLVKPSQSLSLTCTVTGYSITNN...........YAWNWIRQFPGNKLEWMGYIN_......YSGTTSYNPSLKSRISITR........DTSKNQFFLQLNSVTTEDTATYFCVRGYDYFAM-........................DYWGQGTSVTVSS LIGHT CHAIN

QIVLTQSPAIMSASLGDRVTMTCTASSSVSS.-...-SYLHWYQQKPGSSPKLWIYST...-SNLASGVPARFSGSGS.-GTSYSLTISSMEAEDAATYYCHQFHRS....-LTFGSGTKLEIK

Synthesise query IF sequences for every CDR conformation to be predicted

CDR-L1 QITINRESQAVN-.----SSVAYLIYAASGSGTDFYQSSASP-SFA

CDR-L3 SYVLTQSAEALSN---.--QYAYYLIYKDTKTGTVYYQSADSSGD-.-YVFGGYTWIHGLEYLGYVTNYNPSLAEWAEDGHVWFGNAF

CDR-HI SNRFQVQLQQPLKASGYTFTS-.--.-.--.-DWIHWIGEIIP..-.--SRNLADKSSSTAFMYYARERGDGYVGYF

CDR-H2 ASFSN-.--..-.-.-HDVHWMGWMSH-..-.-EGDKTGLAQQTITRDSGASTVYSW

CDR-H3base

NYVPKLSRYLSYFLYNVLWGYEREGNN--DCB

2 Load DCP signatures and Target IF sets of each available cluster in CDR/Length

eg. CDR-L1/12-residues:
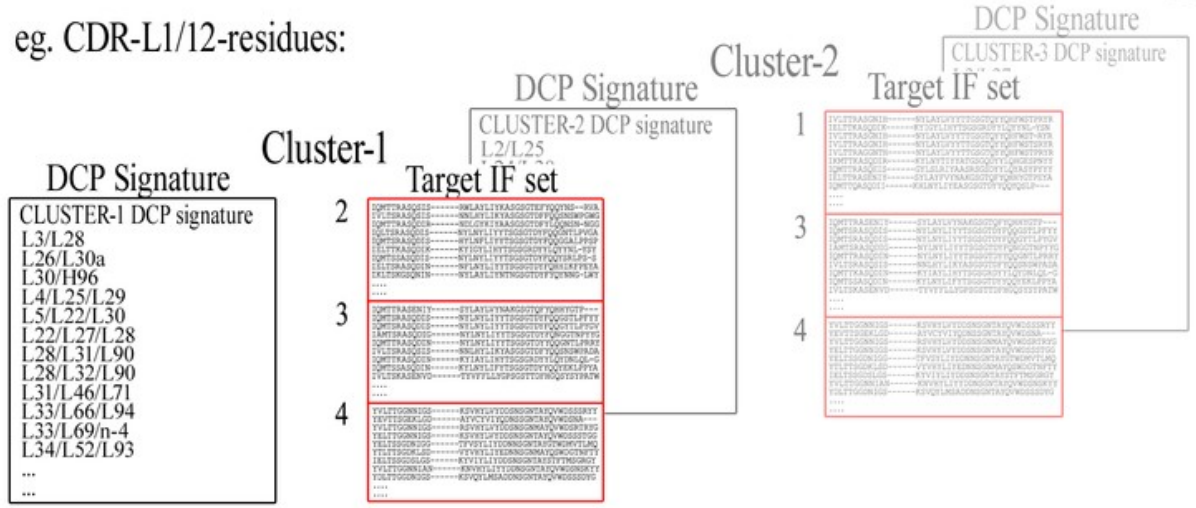

Target IF set

3 Obtain cluster score:

a) read signature signals

b) construct query and target fragment sequences

c) compare query and target sequence fragments for disjointness

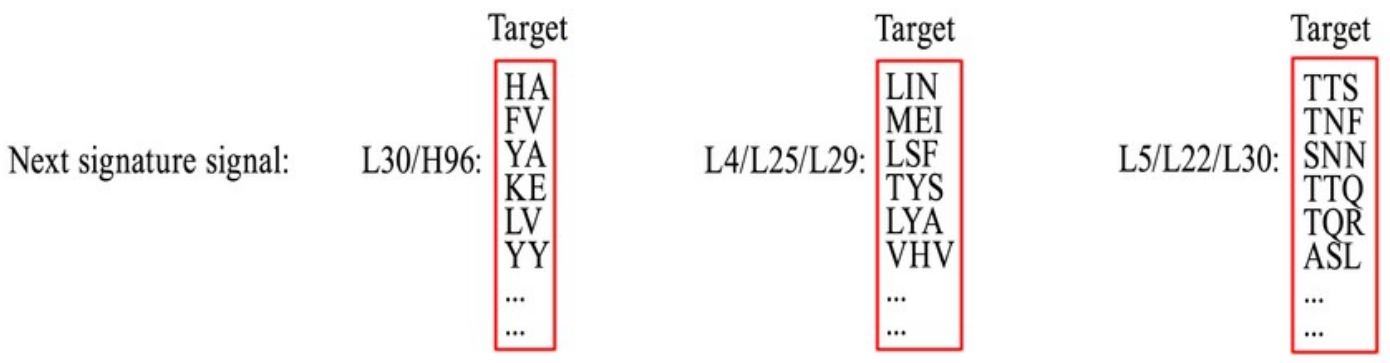

VS.

NS

Query

Score: vs.

TEV

Query

Disjoint?

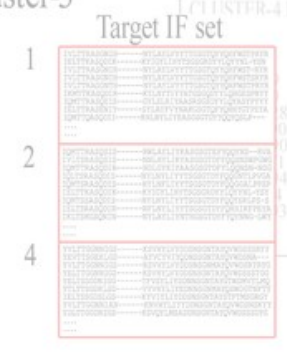

vs.

INN

Query

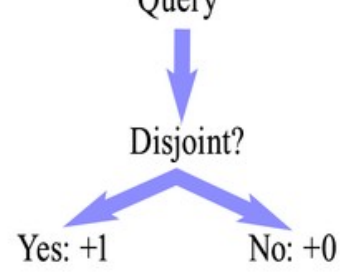

4 When all signature signals are examined for this cluster, store final score, proceed with next cluster

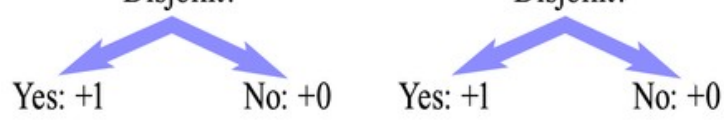

5 Predicted conformation has the best signature matching score ratio: $R=\{$ disjoint/total signature signals $\}$ 


\section{Table 1 (on next page)}

DCP terms and definitions.

A list of terms that were used for the formulation of the DCP method and their definitions. 


\begin{tabular}{|c|c|}
\hline Interaction Frame (IF) & $\begin{array}{l}\text { A list of } F v \text { positions that are found in the neighbourhood of the } \\
\text { examined } C D R \text {, its residues included. }\end{array}$ \\
\hline IF sequence & $\begin{array}{l}\text { A sequence of residues derived from an antibody's Fv that } \\
\text { correspond to the positions included in the IF. }\end{array}$ \\
\hline Query IF sequence set & $\begin{array}{l}\text { A group of non-redundant IF sequences from all members of } \\
\text { the cluster that is being profiled. }\end{array}$ \\
\hline Target IF sequence set & $\begin{array}{l}\text { A group of non-redundant IF sequences from the members of } \\
\text { all clusters in the examined length, excluding the cluster that is } \\
\text { being profiled. }\end{array}$ \\
\hline IF fragment & $\begin{array}{l}\text { A singlet or a non-necessarily consecutive combination of IF } \\
\text { positions (couplet/triplet/quadruplet/etc.). }\end{array}$ \\
\hline IF fragment sequence & The corresponding sequence of residues in an IF fragment. \\
\hline Query/Target fragment sequence & $\begin{array}{l}\text { IF fragment sequences from the Query/Target IF sequence } \\
\text { sets form Query/Target fragment sequences, respectively. }\end{array}$ \\
\hline Signature signal & $\begin{array}{l}\text { An IF fragment that presents disjoint IF fragment sequences } \\
\text { between Query and Target sets. }\end{array}$ \\
\hline DCP signature & $\begin{array}{l}\text { The complete set of signature signals that are consequently } \\
\text { used for the prediction of a given cluster. }\end{array}$ \\
\hline
\end{tabular}

Table 1: A list of terms that were used for the formulation of the DCP method and their definitions. 


\section{Table 2 (on next page)}

Canonical positions.

Table showing the canonical positions per CDR/length, used for CDR conformation prediction by canonical templates. 


\begin{tabular}{|l|l|}
\hline \multicolumn{1}{|c|}{ CDR/Length } & \multicolumn{1}{c|}{ Canonical positions } \\
\hline CDR-L1/11 & L2 L4 L25 L26 L28 L29 L30 L33 L34 L36 L46 L49 L51 L71 L90 L93 \\
\hline CDR-L1/12 & L2 L4 L25 L29 L33 L71 L90 L91 L93 \\
\hline CDR-L1/13 & L4 L25 L29 L30 L33 L66 L71 \\
\hline CDR-L1/14 & L4 L25 L29 L30 L31 L33 L66 L71 L90 \\
\hline CDR-L1/15 & L2 L4 L24 L25 L26 L28 L29 L30 L30c L33 L34 L51 L71 L90 L92 L93 \\
\hline CDR-L1/16 & L2 L4 L25 L26 L27 L29 L30a L30b L30c L30d L32 L33 L34 L51 L71 L90 L92 L93 \\
\hline CDR-L3/8 & L36 L89 L90 L91 L94 L95 L97 L98 \\
\hline CDR-L3/9 & L2 L3 L4 L28 L30 L31 L32 L33 L89 L90 L91 L92 L93 L94 L95 L96 L97 L98 H47 \\
\hline CDR-L3/10 & L4 L32 L36 L89 L90 L91 L92 L95a L96 L97 L98 H47 \\
\hline CDR-H1/13 & H2 H4 H20 H24 H26 H29 H32 H33 H34 H35 H48 H51 H69 H78 H80 H90 H94 H102 \\
\hline CDR-H1/15 & H20 H24 H26 H28 H29 H34 H48 H53 H78 H80 H94 \\
\hline CDR-H2/9 & H47 H51 H55 H59 H69 H71 \\
\hline CDR-H2/10 & H33 H47 H50 H51 H52 H53 H54 H55 H56 H58 H59 H69 H71 H78 \\
\hline CDR-H2/12 & L94 H47 H50 H51 H54 H55 H59 H69 H71 H78 \\
\hline
\end{tabular}

Table 2: Table showing the canonical positions per CDR/length, used for CDR conformation prediction by canonical templates. 


\section{Table 3 (on next page)}

Dataset naming and usage.

Summary of experiments performed, explaining the usage of datasets in each phase. 


\begin{tabular}{|c|c|c|c|}
\hline \multirow[b]{2}{*}{ Dataset } & \multicolumn{3}{|c|}{ Usage } \\
\hline & $\begin{array}{l}\text { Phase } 1 \text { - initial } \\
\text { evaluation }\end{array}$ & \multicolumn{2}{|c|}{ Phase 2 - post-evaluation re-updating } \\
\hline clustering set & $\begin{array}{c}\text { - DCP training } \\
\text { - Canonical templates' } \\
\text { updating }\end{array}$ & \multicolumn{2}{|c|}{$\begin{array}{l}\text { - DCP training } \\
\text { templates' updating }\end{array}$} \\
\hline \multirow{2}{*}{ blind set } & $\begin{array}{c}\text { subset } 1 \text { ("validation } \\
\text { set") }\end{array}$ & $\begin{array}{c}\text { - DCP validation } \\
\text { - Canonical templates' } \\
\text { testing - } \\
\text { Sequence rules' } \\
\text { testing }\end{array}$ & $\begin{array}{c}\text { - DCP training } \\
\text { - Canonical templates' } \\
\text { updating }\end{array}$ \\
\hline & $\begin{array}{l}\text { subset } 2 \text { ("test } \\
\text { set") }\end{array}$ & $\begin{array}{c}\text { - DCP testing } \\
\text { - Canonical templates' } \\
\text { testing - } \\
\text { Sequence rules' } \\
\text { testing }\end{array}$ & $\begin{array}{c}\text { - DCP testing } \\
\text { - Canonical templates' } \\
\text { testing }\end{array}$ \\
\hline
\end{tabular}

Table 3: Summary of experiments performed, explaining the usage of datasets in each phase. 


\section{Table 4 (on next page)}

Interaction Frames that resulted in the construction of the most accurate DCP signatures, and their respective CDR neighbourhood radius.

Notations ' $E$ ', ' $K$ ' and ' $K+$ ', at the end of the CDR-H3-base Interaction Frame, refer to the $\beta$ hairpin type that is favoured at the CDR-H3 apex, depending on the formation of an Extended $(\mathrm{E})$, Kinked $(\mathrm{K})$ and Kinked with double-bulged base $(\mathrm{K}+)$. 


\begin{tabular}{|c|c|c|}
\hline CDR & Interaction Frames & $\begin{array}{l}\text { CDR } \\
\text { Neighbourhood } \\
\text { radius }(\AA)\end{array}$ \\
\hline CDR-L1 & $\begin{array}{l}\text { L2 L3 L4 L5 L22 L24 L25 L26 L27 L28 L29 L30 L30a L30b L30c L30d } \\
\text { L30e L30f L31 L32 L33 L34 L36 L46 L48 L49 L50 L51 L52 L66 L67 L68 } \\
\text { L69 L70 L71 L87 L89 L90 L91 L92 L93 L94 L95 H96 n-4 n-3 }\end{array}$ & 6 \\
\hline CDR-L2 & $\begin{array}{l}\text { L30 L30a L30b L30c L30d L30e L30f L31 L32 L33 L34 L46 L47 L48 L49 } \\
\text { L50 L51 L51a L51b L51c L51d L52 L53 L54 L55 L56 L57 L58 L59 L60 } \\
\text { L61 L62 L63 L64 L65 L66 L67 L71 L72 L91 H32 H101 H102 n-5 n-4 n-3 }\end{array}$ & 4 \\
\hline CDR-L3 & $\begin{array}{c}\text { L1 L2 L3 L4 L27 L28 L29 L30 L30a L30b L30c L30d L30e L30f L31 L32 } \\
\text { L33 L34 L36 L49 L50 L87 L89 L90 L91 L92 L93 L94 L95 L95a L95b } \\
\text { L95c L95d L96 L97 L98 L99 H35 H45 H46 H47 H50 H58 H59 H60 H61 } \\
\text { H95 n-6 n-5 n-4 n-3 n-2 }\end{array}$ & 4 \\
\hline CDR-H1 & $\begin{array}{c}\text { L91 L92 L93 L96 H1 H2 H3 H4 H5 H6 H7 H20 H23 H24 H25 H26 H27 } \\
\text { H28 H29 H30 H31 H31a H31b H31c H31d H31e H31f H31g H31h H31i } \\
\text { H31j H31k H32 H33 H34 H35 H47 H48 H49 H50 H51 H52 H52a H52b } \\
\text { H52c H52d H52e H52f H53 H56 H58 H69 H71 H72 H73 H74 H75 H76 } \\
\text { H77 H78 H79 H80 H90 H91 H93 H94 H95 H96 H97 H98 H99 H100 } \\
\text { H102 n-4 n-3 n-2 }\end{array}$ & 8 \\
\hline CDR-H2 & $\begin{array}{c}\text { H24 H28 H29 H30 H31 H31a H31b H31c H31d H31e H31f H31g H31h } \\
\text { H31i H31j H31k H32 H33 H34 H35 H47 H48 H49 H50 H51 H52 H52a } \\
\text { H52b H52c H52d H52e H52f H53 H54 H55 H56 H57 H58 H59 H60 H61 } \\
\text { H64 H68 H69 H70 H71 H72 H73 H74 H75 H76 H77 H78 H79 L94 L96 }\end{array}$ & 6 \\
\hline $\begin{array}{l}\text { CDR-H3- } \\
\text { base }\end{array}$ & $\begin{array}{l}\text { L34 L36 L43 L44 L45 L46 L49 L55 L87 L89 L91 L96 L98 H4 H27 H35 } \\
\text { H37 H45 H47 H49 H91 H93 H94 H95 H96 H101 H102 n-3 n-2 E K K }\end{array}$ & 4 \\
\hline
\end{tabular}

Table 4: Interaction Frames that resulted in the construction of the most accurate DCP signatures, and their respective CDR neighbourhood radius. Notations ' $E$ ', ' $K$ ' and ' $\mathrm{K}^{+}$', at the end of the CDR-H3-base Interaction Frame, refer to the $\beta$-hairpin type that is favoured at the CDR-H3 apex, depending on the formation of an Extended (E), Kinked $(\mathrm{K})$ and Kinked with double-bulged base $\left(\mathrm{K}^{+}\right)$. 


\section{Table 5 (on next page)}

Individual accuracy percentages per experiment in CDR-L1 and -L3, excluding nonpredictable (novel) conformations.

The previously acquired clustering set was used for initial DCP training and canonical templates' updating. The newly downloaded blind dataset was divided in two subsets: for DCP, subset 1 was used for parameter validation ("validation set"), while subset 2 was used for evaluation ("test set"). Both subsets were used for evaluation of canonical templates, as no parameterisation was necessary, however the terms "validation" and "test" were retained for the two subsets for disambiguation and in order to allow direct comparisons. In postevaluation Phase-2, the validation set was merged to the clustering set for DCP re-training and canonical templates' re-updating. Updated methods were then evaluated on the test set that remained blind, but also were applied for retro-prediction on the validation set. 


\begin{tabular}{|c|c|c|c|c|c|c|c|}
\hline \multicolumn{8}{|c|}{ CDR-L1 predictions } \\
\hline $\begin{array}{l}\text { Phase-1 Initial } \\
\text { DCP signatures }\end{array}$ & $\begin{array}{l}\text { Phase-2 Updated } \\
\text { DCP signatures }\end{array}$ & $\begin{array}{l}\text { Phase-1 Initial } \\
\text { canonical } \\
\text { templates }\end{array}$ & \multicolumn{5}{|c|}{ Phase-2 Updated canonical templates } \\
\hline $\begin{array}{l}\text { Training: } \\
\text { clustering set } \\
\text { Evaluation: } \\
\text { validation set }\end{array}$ & 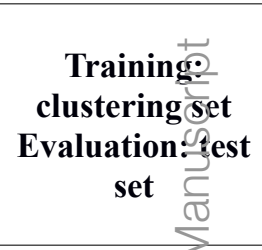 & $\begin{array}{c}\text { Training: } \\
\text { clustering+validati } \\
\text { on sets } \\
\text { Evaluation: } \\
\text { validation set }\end{array}$ & $\begin{array}{c}\text { Training: } \\
\text { clustering+validati } \\
\text { on sets } \\
\text { Evaluation: test } \\
\text { set }\end{array}$ & $\begin{array}{l}\text { Template } \\
\text { Updating: } \\
\text { clustering set } \\
\text { Evaluation: } \\
\text { validation set }\end{array}$ & $\begin{array}{c}\text { Template } \\
\text { Updating: } \\
\text { clustering set } \\
\text { Evaluation: test } \\
\text { set }\end{array}$ & $\begin{array}{c}\text { Training: } \\
\text { clustering+validati } \\
\text { on sets } \\
\text { Evaluation: } \\
\text { validation set }\end{array}$ & $\begin{array}{c}\text { Template } \\
\text { Updating: } \\
\text { clustering+validati } \\
\text { on sets } \\
\text { Evaluation: test } \\
\text { set } \\
\end{array}$ \\
\hline $99 \%(86 / 87)$ & $99 \%(77 / 78)$ & $100 \%(87 / 87)$ & $98 \%(76 / 78)$ & $92 \%(80 / 87)$ & $96 \%(75 / 78)$ & $98 \%(85 / 87)$ & $96 \%(75 / 78)$ \\
\hline $\begin{array}{c}\text { Cumulative } \\
\text { evaluation on } \\
\text { validation+test } \\
\text { sets }\end{array}$ & $\begin{array}{c}\text { Cumulative } \\
\text { evaluation on } \\
\text { validation+fest } \\
\text { sets }\end{array}$ & $\begin{array}{c}\text { Cumulative } \\
\text { evaluation on } \\
\text { validation+test } \\
\text { sets }\end{array}$ & \multicolumn{5}{|c|}{ Cumulative evaluation on validation+test sets } \\
\hline $99 \%(163 / 165)$ & $99 \%(163 / 165)$ & $94 \%(155 / 165)$ & \multicolumn{5}{|c|}{$97 \%(160 / 165)$} \\
\hline \multicolumn{8}{|c|}{ CDR-L3 predictions } \\
\hline $\begin{array}{l}\text { Phase-1 Initial } \\
\text { DCP signatures }\end{array}$ & $\begin{array}{l}\text { Phase-2 UP ted } \\
\text { DCP sign } ₫ \mathrm{~d} \text { es }\end{array}$ & $\begin{array}{c}\text { Phase-1 Initial } \\
\text { canonical } \\
\text { templates }\end{array}$ & \multicolumn{5}{|c|}{ Phase-2 Updated canonical templates } \\
\hline $\begin{array}{l}\text { Training: } \\
\text { clustering set } \\
\text { Evaluation: } \\
\text { validation set }\end{array}$ & $\begin{array}{c}\text { Training: } \\
\text { clustering set } \\
\text { Evaluation: test } \\
\text { set }\end{array}$ & $\begin{array}{c}\text { Training: } \\
\text { clustering+validati } \\
\text { on sets } \\
\text { Evaluation: } \\
\text { validation set }\end{array}$ & $\begin{array}{c}\text { Training: } \\
\text { clustering+validati } \\
\text { on sets } \\
\text { Evaluation: test } \\
\text { set }\end{array}$ & $\begin{array}{l}\text { Template } \\
\text { Updating: } \\
\text { clustering set } \\
\text { Evaluation: } \\
\text { validation set }\end{array}$ & $\begin{array}{c}\text { Template } \\
\text { Updating: } \\
\text { clustering set } \\
\text { Evaluation: test } \\
\text { set }\end{array}$ & $\begin{array}{c}\text { Training: } \\
\text { clustering+validati } \\
\text { on sets } \\
\text { Evaluation: } \\
\text { validation set }\end{array}$ & $\begin{array}{c}\text { Template } \\
\text { Updating: } \\
\text { clustering+validati } \\
\text { on sets } \\
\text { Evaluation: test } \\
\text { set }\end{array}$ \\
\hline $95 \%(84 / 88)$ & $89 \%(70 / 79)$ & $100 \%(88 / 88)$ & $91 \%(72 / 79)$ & $95 \%(69 / 73)$ & $87 \%(62 / 71)$ & $100 \%(73 / 73)$ & $89 \%(63 / 71)$ \\
\hline $\begin{array}{c}\text { Cumulative } \\
\text { evaluation on } \\
\text { validation+test } \\
\text { sets } \\
\end{array}$ & $\begin{array}{c}\text { Cumulative } \\
\text { evaluation on } \\
\text { validation+test } \\
\text { sets } \\
\end{array}$ & $\begin{array}{c}\text { Cumulative } \\
\text { evaluation on } \\
\text { validation+test } \\
\text { sets } \\
\end{array}$ & \multicolumn{5}{|c|}{ Cumulative evaluation on validation+test sets } \\
\hline $92 \%(154 / 167)$ & $96 \%(160 / 167)$ & $91 \%(131 / 144)$ & \multicolumn{5}{|c|}{$94 \%(136 / 144)$} \\
\hline
\end{tabular}

Table 5: Individual accuracy percentages per experiment in CDR-L1 and -L3, excluding non-predictable (novel) conformations. The previously acquired clustering set was used for initial DCP training and canonical templates' updating. The newly downloaded blind dataset was divided in 
two subsets: for DCP, subset 1 was used for parameter validation ("validation set"), while subset 2 was used for evaluation ("test set"). Both subsets were used for evaluation of canonical templates, as no parameterisation was necessary, however the terms "validation" and "test" were retained for the two subsets for disambiguation and in order to allow direct comparisons. In post-evaluation Phase-2, the validation set was merged to the clustering se remained blind, but also we applied for retro-prediction on the validation set.

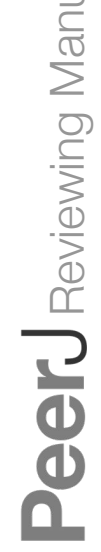




\section{Table 6 (on next page)}

Individual accuracy percentages per experiment in CDR-H1, - $\mathrm{H} 2$ and $-\mathrm{H} 3$, excluding non-predictable (novel) conformations.

Also see notes in Table 5. 


\begin{tabular}{|c|c|c|c|c|c|c|c|}
\hline \multicolumn{8}{|c|}{ CDR-H1 predictions } \\
\hline $\begin{array}{l}\text { Phase- } 1 \text { Initial } \\
\text { DCP signatures }\end{array}$ & $\begin{array}{l}\text { Phase- } 2 \text { Updated } \\
\text { DCP signatures }\end{array}$ & $\begin{array}{c}\text { Phase-1 Initial } \\
\text { canonical } \\
\text { templates }\end{array}$ & \multicolumn{5}{|c|}{ Phase-2 Updated canonical templates } \\
\hline $\begin{array}{l}\text { Training: clustering } \\
\text { set Evaluation: } \\
\text { validation set }\end{array}$ & $\begin{array}{c}\text { Training: } \\
\text { clustering set } \\
\text { Evaluation: test set }\end{array}$ & $\begin{array}{c}\text { Training: } \\
\text { clustering+validatio } \\
\mathrm{n} \text { sets } \\
\text { Evaluation: } \\
\text { validation set }\end{array}$ & $\begin{array}{c}\text { Training: } \\
\text { clustering+validatio } \\
\text { n sets } \\
\text { Evaluation: test set }\end{array}$ & $\begin{array}{l}\text { Template Updating: } \\
\text { clustering set } \\
\text { Evaluation: } \\
\text { validation set }\end{array}$ & $\begin{array}{c}\text { Template } \\
\text { Updating: } \\
\text { clustering set } \\
\text { Evaluation: test set }\end{array}$ & $\begin{array}{c}\text { Training: } \\
\text { clustering+validatio } \\
\mathrm{n} \text { sets } \\
\text { Evaluation: } \\
\text { validation set }\end{array}$ & $\begin{array}{c}\text { Template Updating: } \\
\text { clustering+validatio } \\
\text { n sets } \\
\text { Evaluation: test set }\end{array}$ \\
\hline $96 \%(92 / 96)$ & $95 \%(91 / 9 \%)$ & $100 \%(96 / 96)$ & $96 \%(92 / 96)$ & $79 \%(76 / 96)$ & $83 \%(80 / 96)$ & $83 \%(80 / 96)$ & $85 \%(82 / 96)$ \\
\hline $\begin{array}{c}\text { Cumulative } \\
\text { evaluation on } \\
\text { validation+test sets }\end{array}$ & $\begin{array}{c}\text { Cumulative } \\
\text { evaluation } \\
\text { validation+test sets }\end{array}$ & $\begin{array}{c}\text { Cumulative } \\
\text { evaluation on } \\
\text { validation+test sets }\end{array}$ & \multicolumn{5}{|c|}{ Cumulative evaluation on validation+test sets } \\
\hline $95 \%(183 / 192)$ & $98 \%(188 / 192)$ & $81 \%(156 / 192)$ & \multicolumn{5}{|c|}{$84 \%(162 / 192)$} \\
\hline \multicolumn{8}{|c|}{ CDR-H2 predictions } \\
\hline $\begin{array}{l}\text { Phase- } 1 \text { Initial } \\
\text { DCP signatures }\end{array}$ & $\begin{array}{l}\text { Phase-2 Updated } \\
\text { DCP signatues }\end{array}$ & $\begin{array}{c}\text { Phase-1 Initial } \\
\text { canonical } \\
\text { templates }\end{array}$ & \multicolumn{5}{|c|}{ Phase-2 Updated canonical templates } \\
\hline $\begin{array}{l}\text { Training: clustering } \\
\text { set Evaluation: } \\
\text { validation set }\end{array}$ & 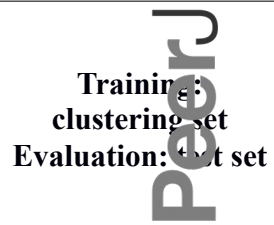 & $\begin{array}{c}\text { Training: } \\
\text { clustering+validatio } \\
\text { n sets } \\
\text { Evaluation: } \\
\text { validation set }\end{array}$ & $\begin{array}{c}\text { Training: } \\
\text { clustering+validatio } \\
\text { n sets } \\
\text { Evaluation: test set }\end{array}$ & $\begin{array}{c}\text { Template Updating: } \\
\text { clustering set } \\
\text { Evaluation: } \\
\text { validation set }\end{array}$ & $\begin{array}{c}\text { Template } \\
\text { Updating: } \\
\text { clustering set } \\
\text { Evaluation: test set }\end{array}$ & $\begin{array}{c}\text { Training: } \\
\text { clustering+validatio } \\
\mathrm{n} \text { sets } \\
\text { Evaluation: } \\
\text { validation set }\end{array}$ & $\begin{array}{c}\text { Template Updating: } \\
\text { clustering+validatio } \\
\text { n sets } \\
\text { Evaluation: test set }\end{array}$ \\
\hline $93 \%(98 / 105)$ & $87 \%(94 / 108)$ & $100 \%(105 / 105)$ & $81 \%(87 / 108)$ & $58 \%(61 / 105)$ & $57 \%(62 / 108)$ & $64 \%(67 / 105)$ & $56 \%(61 / 108)$ \\
\hline $\begin{array}{c}\text { Cumulative } \\
\text { evaluation on } \\
\text { validation+test sets }\end{array}$ & $\begin{array}{c}\text { Cumulative } \\
\text { evaluation on } \\
\text { validation+test sets }\end{array}$ & $\begin{array}{c}\text { Cumulative } \\
\text { evaluation on } \\
\text { validation+test sets }\end{array}$ & \multicolumn{5}{|c|}{ Cumulative evaluation on validation+test sets } \\
\hline $91 \%(193 / 213)$ & $90 \%(192 / 213)$ & $58 \%(123 / 213)$ & \multicolumn{5}{|c|}{$60 \%(128 / 213)$} \\
\hline \multicolumn{8}{|c|}{ CDR-H3-base predictions } \\
\hline $\begin{array}{l}\text { Phase-1 Initial } \\
\text { DCP signatures }\end{array}$ & $\begin{array}{l}\text { Phase-2 Updated } \\
\text { DCP signatures }\end{array}$ & 1999 sequence rules & \multicolumn{5}{|c|}{2007 sequence rules } \\
\hline $\begin{array}{l}\text { Training: clustering } \\
\text { set Evaluation: } \\
\text { validation set }\end{array}$ & $\begin{array}{c}\text { Training: } \\
\text { clustering set } \\
\text { Evaluation: test set }\end{array}$ & $\begin{array}{c}\text { Training: } \\
\text { clustering+validatio } \\
\text { n sets } \\
\text { Evaluation: } \\
\text { validation set }\end{array}$ & $\begin{array}{c}\text { Training: } \\
\text { clustering+validatio } \\
\text { n sets } \\
\text { Evaluation: test set }\end{array}$ & $\begin{array}{l}\text { Evaluation: } \\
\text { validation set }\end{array}$ & Evaluation: test set & $\begin{array}{l}\text { Evaluation: } \\
\text { validation set }\end{array}$ & Evaluation: test set \\
\hline $89 \%(93 / 104)$ & $91 \%(102 / 112)$ & $100 \%(104 / 104)$ & $88 \%(99 / 112)$ & $83 \%(86 / 104)$ & $87 \%(97 / 112)$ & $86 \%(89 / 104)$ & $84 \%(94 / 112)$ \\
\hline $\begin{array}{c}\text { Cumulative } \\
\text { evaluation on } \\
\text { validation+test sets }\end{array}$ & $\begin{array}{c}\text { Cumulative } \\
\text { evaluation on } \\
\text { validation+test sets }\end{array}$ & $\begin{array}{c}\text { Cumulative } \\
\text { evaluation on } \\
\text { validation+test sets }\end{array}$ & \multicolumn{5}{|c|}{ Cumulative evaluation on validation+test sets } \\
\hline
\end{tabular}


\begin{tabular}{|l|l|l|r}
\hline $90 \%(195 / 216)$ & $94 \%(203 / 216)$ & $85 \%(183 / 216)$ & $85 \%(183 / 216)$ \\
\hline
\end{tabular}

$85 \%(183 / 216)$

Table 6: Individual accuracy percentages per experiment in CDR-H1, - $\mathrm{H} 2$ and -H3, excluding non-predictable (novel) conformations. Also see notes in Table 5.

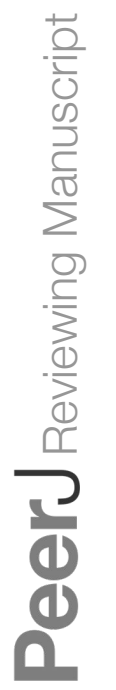




\section{Table 7 (on next page)}

Summary table of Phase-1 prediction results over all test data belonging to non-single cluster lengths, for CDR-L1 and -L3.

Percentages are rounded to the closest unit. Totals for canonical templates in CDR-L3 are marked in italics because they don't include predictions for a length of 11-residues (no template available). For a direct comparison, total accurate predictions for DCP signatures for 8-, 9- and 10-resides CDR-L3 were 133/153 (87\%). Totals include novel conformations. 


\begin{tabular}{|c|c|c|c|c|c|c|c|c|c|}
\hline & $\begin{array}{c}\text { DCP } \\
\text { signatures }\end{array}$ & & $\begin{array}{l}\text { Canonical } \\
\text { Templates }\end{array}$ & & & & & & \\
\hline CDR/Length & $\begin{array}{l}\text { Accurately } \\
\text { predicted } \\
\text { conformation }\end{array}$ & $\begin{array}{l}\text { Uncertain } \\
\text { predictions }\end{array}$ & $\begin{array}{c}\text { False } \\
\text { predictions }\end{array}$ & $\begin{array}{c}\text { Novel } \\
\text { conformatio } \\
\text { ns }\end{array}$ & $\begin{array}{l}\text { Accurately } \\
\text { predicted } \\
\text { conformation } \\
\text { s }\end{array}$ & $\begin{array}{l}\text { Uncertain } \\
\text { predictions }\end{array}$ & $\begin{array}{l}\text { False } \\
\text { predictions }\end{array}$ & $\begin{array}{l}\text { New CDR } \\
\text { sequences in } \\
\text { test set } \\
\text { (not present } \\
\text { in training } \\
\text { set) }\end{array}$ & $\begin{array}{c}\text { Unique CDR } \\
\text { sequences in } \\
\text { training set }\end{array}$ \\
\hline CDR-L1-11 & $\begin{array}{l}977^{\frac{11}{11}} 07 \\
(9 \% \%)\end{array}$ & 0/105 & $0 / 107$ & $10 / 107(9 \%)$ & $\begin{array}{l}93 / 107 \\
(87 \%) \\
\end{array}$ & 4/107 (4\%) & $0 / 107$ & $\begin{array}{c}68 / 107 \\
(64 \%) \\
\end{array}$ & 177 \\
\hline CDR-L1-12 & $11 / 14 \vec{\sigma} 79 \%)$ & $0 / 14$ & $2 / 14(14 \%)$ & $1 / 14(7 \%)$ & $8 / 14(57 \%)$ & $4 / 14(29 \%)$ & $\begin{array}{l}1 / 14 \\
(7 \%)\end{array}$ & 11/14 (79\%) & 25 \\
\hline CDR-L1-13 & 16/1خ94\%) & $0 / 17$ & $0 / 17$ & $1 / 17(6 \%)$ & $16 / 17(94 \%)$ & $0 / 17$ & $0 / 17$ & $15 / 17(88 \%)$ & 26 \\
\hline CDR-L1-14 & $\begin{array}{c}10 \operatorname{0in} 0 \\
(10 \%)\end{array}$ & $0 / 10$ & $0 / 10$ & $0 / 10$ & $\begin{array}{c}10 / 10 \\
(100 \%)\end{array}$ & $0 / 10$ & $0 / 10$ & $6 / 10(60 \%)$ & 26 \\
\hline CDR-L1-15 & 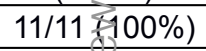 & $0 / 11$ & $0 / 11$ & $0 / 11$ & $10 / 11(91 \%)$ & $1 / 11(9 \%)$ & $0 / 11$ & 9/11 (82\%) & 16 \\
\hline CDR-L1-16 & $\begin{array}{c}18,18 \\
(100 \%)\end{array}$ & $0 / 18$ & $0 / 18$ & $0 / 18$ & $\begin{array}{c}18 / 18 \\
(100 \%)\end{array}$ & $0 / 18$ & $0 / 18$ & $10 / 18(56 \%)$ & 71 \\
\hline \multirow[t]{2}{*}{ Total } & $\begin{array}{l}1631177 \\
(92 \%)\end{array}$ & $0 / 177$ & 2/177 (1\%) & $12 / 177(7 \%)$ & $\begin{array}{c}155 / 177 \\
(88 \%) \\
\end{array}$ & 9/177 (5\%) & $1 / 177(0.5 \%)$ & $\begin{array}{c}119 / 177 \\
(67 \%) \\
\end{array}$ & 341 \\
\hline & sigioures & & $\begin{array}{l}\text { Canonical } \\
\text { Templates } \\
\end{array}$ & & & & & & \\
\hline CDR/Length & $\begin{array}{l}\text { A urately } \\
\text { prunicted } \\
\text { conformation } \\
s\end{array}$ & $\begin{array}{l}\text { Uncertain } \\
\text { predictions }\end{array}$ & $\begin{array}{c}\text { False } \\
\text { predictions }\end{array}$ & $\begin{array}{c}\text { Novel } \\
\text { conformatio } \\
\text { ns }\end{array}$ & $\begin{array}{l}\text { Accurately } \\
\text { predicted } \\
\text { conformation } \\
\text { s }\end{array}$ & $\begin{array}{l}\text { Uncertain } \\
\text { predictions }\end{array}$ & $\begin{array}{l}\text { False } \\
\text { predictions }\end{array}$ & $\begin{array}{c}\text { New CDR } \\
\text { sequences in } \\
\text { test set } \\
\text { (not present } \\
\text { in training } \\
\text { set) }\end{array}$ & $\begin{array}{c}\text { Unique CDR } \\
\text { sequences in } \\
\text { training set }\end{array}$ \\
\hline CDR-L3-8 & $18 / 19(95 \%)$ & $0 / 19$ & $1 / 19(5 \%)$ & $0 / 19$ & $17 / 19(89 \%)$ & $1 / 19(5 \%)$ & $1 / 19(5 \%)$ & $12 / 19(63 \%)$ & 44 \\
\hline CDR-L3-9 & $\begin{array}{c}111 / 119 \\
(93 \%) \\
\end{array}$ & $1 / 119(1 \%)$ & $6 / 119(5 \%)$ & 1/119 (1\%) & $\begin{array}{c}110 / 119 \\
(92 \%) \\
\end{array}$ & $4 / 119(3 \%)$ & $4 / 119(3 \%)$ & $\begin{array}{l}84 / 119 \\
(71 \%) \\
\end{array}$ & 359 \\
\hline CDR-L3-10 & $4 / 15(27 \%)$ & $0 / 15$ & $4 / 15(27 \%)$ & $7 / 15$ (47\%) & $4 / 15(27 \%)$ & $2 / 15(13 \%)$ & $2 / 15(13 \%)$ & 14/15 (93\%) & 26 \\
\hline CDR-L3-11 & 19/25 (76\%) & $0 / 25$ & $1 / 25(4 \%)$ & $5 / 25(20 \%)$ & $\mathrm{N} / \mathrm{A}$ & $\mathrm{N} / \mathrm{A}$ & $\mathrm{N} / \mathrm{A}$ & $23 / 25(92 \%)$ & 36 \\
\hline Total & $\begin{array}{c}152 / 178 \\
(85 \%)\end{array}$ & $1 / 178(1 \%)$ & $12 / 178(7 \%)$ & 13/178 (7\%) & $\begin{array}{c}131 / 153 \\
(86 \%)\end{array}$ & 7/153 (5\%) & 7/153 (5\%) & $\begin{array}{c}133 / 178 \\
(75 \%)\end{array}$ & 465 \\
\hline
\end{tabular}

Table 7: Summary table of Phase-1 prediction results over all test data belonging to non-single cluster lengths, for CDR-L1 and -L3. Percentages are rounded to the closest unit. Totals for canonical templates in CDR-L3 are marked in italics because they don't include predictions for a length 
of 11-residues (no template available). For a direct comparison, total accurate predictions for DCP signatures for 8-, 9- and 10-resides CDR-L3 were 133/153 (87\%). Totals include novel conformations.

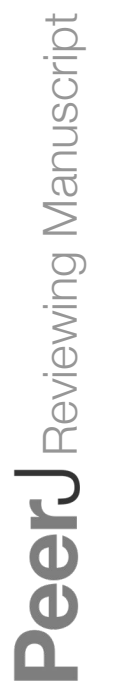




\section{Table 8 (on next page)}

Extended performance measures for major cluster (class-I) predictions in each CDR-L1 and -L3 length (Phase-1).

No canonical templates were available for CDR-L3/11-residues. The asterisk points the fact that clusters in CDR-L3/10-residues are all small, however cluster CDR-L3-10-I was technically considered here for consistency with all other major clusters. 


\begin{tabular}{|c|c|c|c|c|c|c|c|c|}
\hline & \multicolumn{8}{|c|}{ DCP signatures } \\
\hline & $\begin{array}{c}\text { Class-I } \\
\text { prediction }\end{array}$ & \multicolumn{7}{|c|}{ Statistics } \\
\hline $\begin{array}{l}\text { CDR/Lengt } \\
\mathrm{h}\end{array}$ & $\begin{array}{c}\text { True } \\
\text { Positives }\end{array}$ & $\begin{array}{c}\text { True } \\
\text { Negatives }\end{array}$ & $\begin{array}{c}\text { False } \\
\text { Positives }\end{array}$ & $\begin{array}{c}\text { False } \\
\text { Negatives }\end{array}$ & Accuracy & Precision & Recall & $\begin{array}{c}\mathrm{F}- \\
\text { measure }\end{array}$ \\
\hline CDR-L1-11 & 82 & 21 & 4 & 0 & 0,96 & 0,95 & 1,00 & 0,98 \\
\hline CDR-L1-12 & 7 & 5 & 1 & 1 & 0,86 & 0,88 & 0,88 & 0,88 \\
\hline CDR-L1-13 & 18 & 0 & 1 & 0 & 0,95 & 0,95 & 1,00 & 0,97 \\
\hline CDR-L1-14 & 8 & 2 & 0 & 0 & 1,00 & 1,00 & 1,00 & 1,00 \\
\hline CDR-L1-15 & 11 & 0 & 0 & 0 & 1,00 & 1,00 & 1,00 & 1,00 \\
\hline \multirow[t]{3}{*}{ CDR-L1-16 } & 18 & 0 & 0 & 0 & 1,00 & 1,00 & 1,00 & 1,00 \\
\hline & \multicolumn{8}{|c|}{ Canonical templates } \\
\hline & $\begin{array}{c}\text { Class-I } \\
\text { prediction } \\
\mathrm{s}\end{array}$ & \multicolumn{7}{|c|}{ Statistics } \\
\hline $\begin{array}{l}\text { CDR/Lengt } \\
\mathrm{h}\end{array}$ & $\begin{array}{c}\text { True } \\
\text { Positives }\end{array}$ & $\begin{array}{c}\text { True } \\
\text { Negatives }\end{array}$ & $\begin{array}{c}\text { False } \\
\text { Positives }\end{array}$ & $\begin{array}{c}\text { False } \\
\text { Negatives }\end{array}$ & Accuracy & Precision & Recall & $\begin{array}{c}\mathrm{F}- \\
\text { measure }\end{array}$ \\
\hline CDR-L1-11 & 82 & 21 & 4 & 0 & 0,96 & 0,95 & 1,00 & 0,98 \\
\hline CDR-L1-12 & 4 & 5 & 0 & 5 & 0,64 & 1,00 & 0,44 & 0,62 \\
\hline CDR-L1-13 & 18 & 1 & 0 & 0 & 1,00 & 1,00 & 1,00 & 1,00 \\
\hline CDR-L1-14 & 8 & 2 & 0 & 0 & 1,00 & 1,00 & 1,00 & 1,00 \\
\hline CDR-L1-15 & 10 & 0 & 0 & 1 & 0,91 & 1,00 & 0,91 & 0,95 \\
\hline \multirow[t]{3}{*}{ CDR-L1-16 } & 18 & 0 & 0 & 0 & 1,00 & 1,00 & 1,00 & 1,00 \\
\hline & \multicolumn{8}{|c|}{ DCP signatures } \\
\hline & $\begin{array}{c}\text { Class-I } \\
\text { prediction } \\
\mathrm{s}\end{array}$ & \multicolumn{7}{|c|}{ Statistics } \\
\hline $\begin{array}{l}\text { CDR/Lengt } \\
\mathrm{h}\end{array}$ & $\begin{array}{c}\text { True } \\
\text { Positives }\end{array}$ & $\begin{array}{c}\text { True } \\
\text { Negatives }\end{array}$ & $\begin{array}{c}\text { False } \\
\text { Positives }\end{array}$ & $\begin{array}{c}\text { False } \\
\text { Negatives }\end{array}$ & Accuracy & Precision & Recall & $\begin{array}{c}\mathrm{F}- \\
\text { measure }\end{array}$ \\
\hline CDR-L3-8 & 14 & 4 & 1 & 0 & 0,95 & 0,93 & 1,00 & 0,97 \\
\hline CDR-L3-9 & 107 & 4 & 8 & 0 & 0,93 & 0,93 & 1,00 & 0,96 \\
\hline $\begin{array}{l}\text { CDR-L3- } \\
10^{*} \\
\end{array}$ & 1 & 11 & 0 & 3 & 0,80 & 1,00 & 0,25 & 0,40 \\
\hline \multirow[t]{3}{*}{ CDR-L3-11 } & 19 & 1 & 5 & 0 & 0,80 & 0,79 & 1,00 & 0,88 \\
\hline & \multicolumn{8}{|c|}{ Canonical templates } \\
\hline & $\begin{array}{c}\text { Class-I } \\
\text { prediction } \\
\mathrm{s}\end{array}$ & \multicolumn{7}{|c|}{ Statistics } \\
\hline $\begin{array}{l}\text { CDR/Lengt } \\
\mathrm{h}\end{array}$ & $\begin{array}{c}\text { True } \\
\text { Positives }\end{array}$ & $\begin{array}{c}\text { True } \\
\text { Negatives }\end{array}$ & $\begin{array}{c}\text { False } \\
\text { Positives }\end{array}$ & $\begin{array}{c}\text { False } \\
\text { Negatives }\end{array}$ & Accuracy & Precision & Recall & $\begin{array}{c}\mathrm{F}- \\
\text { measure }\end{array}$ \\
\hline CDR-L3-8 & 13 & 4 & 1 & 1 & 0,89 & 0,93 & 0,93 & 0,93 \\
\hline CDR-L3-9 & 105 & 8 & 4 & 2 & 0,95 & 0,96 & 0,98 & 0,97 \\
\hline $\begin{array}{l}\text { CDR-L3- } \\
10^{*}\end{array}$ & 1 & 11 & 0 & 3 & 0,80 & 1,00 & 0,25 & 0,40 \\
\hline CDR-L3-11 & - & - & - & - & - & - & - & - \\
\hline
\end{tabular}

Table 8: Extended performance measures for major cluster (class-I) predictions in each CDR-L1 and -L3 length (Phase-1). No canonical templates were available for CDR-L3/11-residues. The asterisk points the fact that clusters in CDR-L3/10-residues are all small, however cluster CDR-L3-10-I was technically considered here for consistency with all other major clusters. 


\section{Table 9 (on next page)}

Summary table of Phase-1 prediction results over all test data belonging to non-uniquecluster lengths, for CDR-H1 and $-\mathrm{H} 2$.

Totals include novel conformations. 


\begin{tabular}{|c|c|c|c|c|c|c|c|c|c|}
\hline & $\begin{array}{c}\mathrm{DCP} \\
\text { signatures }\end{array}$ & & $\begin{array}{l}\text { Canonical } \\
\text { Templates }\end{array}$ & & & & & & \\
\hline CDR/Length & $\begin{array}{c}\text { Accurately } \\
\text { predicted } \\
\text { conformatio } \\
\text { ns }\end{array}$ & 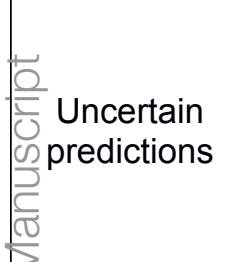 & $\begin{array}{c}\text { False } \\
\text { predictions }\end{array}$ & $\begin{array}{c}\text { Novel } \\
\text { conformati } \\
\text { ons }\end{array}$ & $\begin{array}{c}\text { Accurately } \\
\text { predicted } \\
\text { conformatio } \\
\text { ns }\end{array}$ & $\begin{array}{l}\text { Uncertain } \\
\text { predictions }\end{array}$ & $\begin{array}{c}\text { False } \\
\text { predictions }\end{array}$ & $\begin{array}{c}\text { New CDR } \\
\text { sequences } \\
\text { in test set } \\
\text { (not present } \\
\text { in training } \\
\text { set) }\end{array}$ & $\begin{array}{c}\text { Unique CDR } \\
\text { sequences } \\
\text { in training } \\
\text { set }\end{array}$ \\
\hline CDR-H1-13 & $\begin{array}{c}178 / 201 \\
(89 \%)\end{array}$ & 0/201 & $8 / 201(4 \%)$ & $16 / 201(8 \%)$ & $\begin{array}{c}153 / 201 \\
(76 \%)\end{array}$ & $\begin{array}{c}24 / 201 \\
(12 \%)\end{array}$ & $8 / 201(4 \%)$ & $\begin{array}{c}135 / 201 \\
(67 \%)\end{array}$ & 419 \\
\hline CDR-H1-15 & $5 / 9(56 \%)$ & $0 / 9$ & $2 / 9(22 \%)$ & $2 / 9(22 \%)$ & $3 / 9(33 \%)$ & $2 / 9(22 \%)$ & $2 / 9(22 \%)$ & $8 / 9(89 \%)$ & 27 \\
\hline \multirow[t]{2}{*}{ Total } & $\begin{array}{c}183 / 210(87 \\
\%)\end{array}$ & id $0 / 210$ & $10 / 210(5 \%)$ & $18 / 210(9 \%)$ & $\begin{array}{c}156 / 210 \\
(74 \%)\end{array}$ & $\begin{array}{c}26 / 210 \\
(12 \%)\end{array}$ & $10 / 210(5 \%)$ & $\begin{array}{c}143 / 210 \\
(68 \%)\end{array}$ & 446 \\
\hline & $\begin{array}{c}\text { DCP } \\
\text { signatures }\end{array}$ & D & $\begin{array}{l}\text { Canonical } \\
\text { Templates }\end{array}$ & & & & & & \\
\hline CDR/Length & $\begin{array}{l}\text { Accurately } \\
\text { predicted } \\
\text { conformatic } \\
\text { ns }\end{array}$ & $\begin{array}{l}\text { Uncertain } \\
\text { predictions }\end{array}$ & $\begin{array}{c}\text { False } \\
\text { predictions }\end{array}$ & $\begin{array}{c}\text { Novel } \\
\text { conformati } \\
\text { ons }\end{array}$ & $\begin{array}{c}\text { Accurately } \\
\text { predicted } \\
\text { conformatio } \\
\text { ns }\end{array}$ & $\begin{array}{l}\text { Uncertain } \\
\text { predictions }\end{array}$ & $\begin{array}{c}\text { False } \\
\text { predictions }\end{array}$ & $\begin{array}{c}\text { New CDR } \\
\text { sequences } \\
\text { in test set } \\
\text { (not present } \\
\text { in training } \\
\text { set) }\end{array}$ & $\begin{array}{c}\text { Unique CDR } \\
\text { sequences } \\
\text { in training } \\
\text { set }\end{array}$ \\
\hline CDR-H2-9 & $\begin{array}{c}41 / 41 \\
(100 \%)\end{array}$ & $0 / 41$ & $0 / 41$ & $0 / 41$ & $27 / 41(66 \%)$ & $14 / 41(34 \%)$ & $0 / 41$ & $30 / 41(73 \%)$ & 117 \\
\hline CDR-H2-10 & $\begin{array}{c}145 / 168 \\
(86 \%)\end{array}$ & $6 / 168(4 \%)$ & 14/168 (8\%) & $3 / 168(2 \%)$ & $\begin{array}{c}89 / 168 \\
(53 \%)\end{array}$ & $\begin{array}{c}60 / 168 \\
(36 \%)\end{array}$ & $\begin{array}{l}16 / 168 \\
(10 \%)\end{array}$ & $\begin{array}{c}128 / 168 \\
(76 \%)\end{array}$ & 350 \\
\hline CDR-H2-12 & $7 / 8(88 \%)$ & $0 / 8$ & $0 / 8$ & $1 / 8(13 \%)$ & $7 / 8(88 \%)$ & $0 / 8$ & $0 / 8$ & $4 / 8(50 \%)$ & 39 \\
\hline Total & $\begin{array}{c}193 / 217 \\
(89 \%)\end{array}$ & $6 / 217(3 \%)$ & $14 / 217(6 \%)$ & $4 / 217(2 \%)$ & $\begin{array}{c}123 / 217 \\
(57 \%)\end{array}$ & $\begin{array}{c}74 / 217 \\
(34 \%)\end{array}$ & $16 / 217(7 \%)$ & $\begin{array}{c}162 / 217 \\
(75 \%)\end{array}$ & 506 \\
\hline
\end{tabular}

Table 9: Summary table of Phase-1 prediction results over all test data belonging to non-unique-cluster lengths, for CDR-H1 and -H2. Totals include novel conformations. 


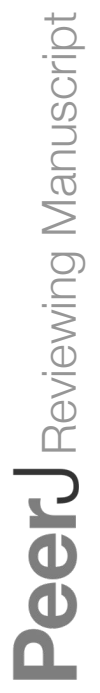

PeerJ reviewing PDF | (v2014:03:1771:1:1:NEW 26 May 2014) 


\section{Table 10 (on next page)}

Extended performance measures for major cluster (class-I) predictions in each CDR-H1 and $-\mathrm{H} 2$ length (Phase 1).

Extended performance measures for major cluster (class-I) predictions in each CDR-H1 and $-\mathrm{H} 2$ length (Phase 1). 


\begin{tabular}{|c|c|c|c|c|c|c|c|c|}
\hline & \multicolumn{8}{|c|}{ DCP signatures } \\
\hline & \multirow{2}{*}{$\begin{array}{l}\begin{array}{l}\text { Class-I } \\
\text { predictio } \\
\text { ns }\end{array} \\
\begin{array}{l}\text { True } \\
\text { Positives }\end{array}\end{array}$} & \multicolumn{7}{|l|}{ Statistics } \\
\hline $\begin{array}{l}\text { CDR/Leng } \\
\text { th }\end{array}$ & & $\begin{array}{l}\text { True } \\
\text { Negative } \\
\mathrm{s}\end{array}$ & $\begin{array}{l}\text { False } \\
\text { Positives }\end{array}$ & $\begin{array}{l}\text { False } \\
\text { Negative } \\
\mathrm{s}\end{array}$ & Accuracy & Precision & Recall & $\begin{array}{l}\mathrm{F} \text { - } \\
\text { measure }\end{array}$ \\
\hline $\begin{array}{l}\text { CDR-H1- } \\
130\end{array}$ & 173 & 6 & 21 & 1 & 0,89 & 0,89 & 0,99 & 0,94 \\
\hline $\begin{array}{l}\text { CDR-41- } \\
15\end{array}$ & 4 & 1 & 4 & 0 & 0,56 & 0,50 & 1,00 & 0,67 \\
\hline$\overline{\bar{U}}$ & \multicolumn{8}{|c|}{ Canonical templates } \\
\hline ת & $\begin{array}{l}\text { Class-I } \\
\text { predictio } \\
\text { ns }\end{array}$ & \multicolumn{7}{|l|}{ Statistics } \\
\hline $\begin{array}{l}\text { CDR } \\
\text { th }\end{array}$ & $\begin{array}{l}\text { True } \\
\text { Positives }\end{array}$ & $\begin{array}{l}\text { True } \\
\text { Negative } \\
\mathrm{s}\end{array}$ & $\begin{array}{l}\text { False } \\
\text { Positives }\end{array}$ & $\begin{array}{l}\text { False } \\
\text { Negative } \\
\mathrm{s}\end{array}$ & Accuracy & Precision & Recall & $\begin{array}{l}\mathrm{F} \text { - } \\
\text { measure }\end{array}$ \\
\hline $\begin{array}{l}\text { CDRAH1- } \\
13 \text { - }\end{array}$ & 153 & 7 & 20 & 21 & 0,80 & 0,88 & 0,88 & 0,88 \\
\hline $\begin{array}{l}\text { CDR-H1- } \\
\frac{15}{15}\end{array}$ & 3 & 2 & 3 & 1 & 0,56 & 0,50 & 0,75 & 0,60 \\
\hline 2 & \multicolumn{8}{|c|}{ DCP signatures } \\
\hline (c) & $\begin{array}{l}\text { Class-I } \\
\text { predictio } \\
\text { ns }\end{array}$ & \multicolumn{7}{|l|}{ Statistics } \\
\hline $\begin{array}{l}\text { (1/lleng } \\
\text { th }\end{array}$ & $\begin{array}{l}\text { True } \\
\text { Positives }\end{array}$ & $\begin{array}{l}\text { True } \\
\text { Negative } \\
\mathrm{s}\end{array}$ & $\begin{array}{l}\text { False } \\
\text { Positives }\end{array}$ & $\begin{array}{l}\text { False } \\
\text { Negative } \\
\mathrm{s}\end{array}$ & Accuracy & Precision & Recall & $\begin{array}{l}\mathrm{F} \text { - } \\
\text { measure }\end{array}$ \\
\hline CDR-H2-9 & 41 & 0 & 0 & 0 & 1,00 & 1,00 & 1,00 & 1,00 \\
\hline $\begin{array}{l}\text { CDR-H2- } \\
10\end{array}$ & 103 & 45 & 4 & 16 & 0,88 & 0,96 & 0,87 & 0,91 \\
\hline \multirow[t]{3}{*}{$\begin{array}{l}\text { CDR-H2- } \\
12 \\
\end{array}$} & 7 & 0 & 1 & 0 & 0,88 & 0,88 & 1,00 & 0,93 \\
\hline & \multicolumn{8}{|c|}{ Canonical templates } \\
\hline & $\begin{array}{l}\text { Class-I } \\
\text { predictio } \\
\text { ns }\end{array}$ & \multicolumn{7}{|l|}{ Statistics } \\
\hline $\begin{array}{l}\text { CDR/Leng } \\
\text { th }\end{array}$ & $\begin{array}{l}\text { True } \\
\text { Positives }\end{array}$ & $\begin{array}{l}\text { True } \\
\text { Negative } \\
\mathrm{s}\end{array}$ & $\begin{array}{l}\text { False } \\
\text { Positives }\end{array}$ & $\begin{array}{l}\text { False } \\
\text { Negative } \\
\mathrm{s}\end{array}$ & Accuracy & Precision & Recall & $\begin{array}{l}\mathrm{F} \text { - } \\
\text { measure }\end{array}$ \\
\hline CDR-H2-9 & 27 & 0 & 0 & 14 & 0,66 & 1,00 & 0,66 & 0,79 \\
\hline $\begin{array}{l}\text { CDR-H2- } \\
10\end{array}$ & 74 & 43 & 6 & 45 & 0,70 & 0,93 & 0,62 & 0,74 \\
\hline $\begin{array}{l}\text { CDR-H2- } \\
12\end{array}$ & 7 & 0 & 1 & 0 & 0,88 & 0,88 & 1,00 & 0,93 \\
\hline
\end{tabular}

Table 10: Extended performance measures for major cluster (class-I) predictions in each CDR-H1 and -H2 length (Phase 1). 


\section{Table 11 (on next page)}

Summary table of Phase-1 prediction results for the CDR-H3-base conformation over all test data.

Summary table of Phase-1 prediction results for the CDR-H3-base conformation over all test data. 


\begin{tabular}{|c|c|c|c|c|c|c|}
\hline \multirow{3}{*}{$\begin{array}{c}\text { CDR-H3- } \\
\text { base } \\
\text { conformatio } \\
n\end{array}$} & $\begin{array}{c}\mathrm{DCP} \\
\text { sianatures }\end{array}$ & & \multicolumn{4}{|c|}{ H3-rules, 2007 edition } \\
\hline & $\begin{array}{c}\text { Accurately } \\
\text { predicted } \\
\text { conformatio } \\
\text { ns }\end{array}$ & $\begin{array}{c}\text { False } \\
\text { predictions }\end{array}$ & $\begin{array}{c}\text { Accurately } \\
\text { predicted } \\
\text { conformatio } \\
\text { ns }\end{array}$ & $\begin{array}{l}\text { False } \\
\text { predictions }\end{array}$ & $\begin{array}{c}\text { Accurately } \\
\text { predicted } \\
\text { conformatio } \\
\text { ns }\end{array}$ & $\begin{array}{c}\text { False } \\
\text { predictions }\end{array}$ \\
\hline & $\begin{array}{c}195 / 216(90 \\
\%)\end{array}$ & $\begin{array}{c}21 / 216 \\
(10 \%)\end{array}$ & $\begin{array}{c}183 / 216 \\
(85 \%)\end{array}$ & $\begin{array}{c}33 / 216 \\
(15 \%)\end{array}$ & $\begin{array}{c}183 / 216 \\
(85 \%)\end{array}$ & $\begin{array}{l}33 / 216 \\
(15 \%)\end{array}$ \\
\hline
\end{tabular}

Table 11: Summary table of Phase-1 prediction results for the CDR-H3-base conformation over all test data. 


\section{Table 12(on next page)}

Extended performance measures for Kinked base predictions in CDR-H3 (Phase 1).

Extended performance measures for Kinked base predictions in CDR-H3 (Phase 1). 


\begin{tabular}{|c|c|c|c|c|c|c|c|c|}
\hline \multirow{12}{*}{$\begin{array}{l}\text { CDR-H3, } \\
\text { kinked } \\
\text { base } \\
\text { conformat } \\
\text { ion }\end{array}$} & \multicolumn{8}{|c|}{ DCP signatures } \\
\hline & $\begin{array}{c}\text { Class-I } \\
\text { prediction }\end{array}$ & \multicolumn{7}{|c|}{ Statistics } \\
\hline & $\begin{array}{c}\text { True } \\
\text { Positives }\end{array}$ & $\begin{array}{c}\text { True } \\
\text { Negatives }\end{array}$ & $\begin{array}{c}\text { False } \\
\text { Positives }\end{array}$ & $\begin{array}{c}\text { False } \\
\text { Negatives }\end{array}$ & Accuracy & Precision & Recall & $\begin{array}{c}\mathrm{F}- \\
\text { measure }\end{array}$ \\
\hline & 191 & 4 & 13 & 8 & 0,90 & 0,94 & 0,96 & 0,95 \\
\hline & \multicolumn{8}{|c|}{ H3-rules, 1999 edition } \\
\hline & $\begin{array}{c}\text { Class-I } \\
\text { prediction } \\
\mathrm{s} \\
\end{array}$ & \multicolumn{7}{|c|}{ Statistics } \\
\hline & $\begin{array}{c}\text { True } \\
\text { Positives }\end{array}$ & $\begin{array}{c}\text { True } \\
\text { Negatives }\end{array}$ & $\begin{array}{c}\text { False } \\
\text { Positives }\end{array}$ & $\begin{array}{c}\text { False } \\
\text { Negatives }\end{array}$ & Accuracy & Precision & Recall & $\begin{array}{c}\mathrm{F}- \\
\text { measure }\end{array}$ \\
\hline & 182 & 1 & 15 & 18 & 0,85 & 0,92 & 0,91 & 0,92 \\
\hline & \multicolumn{8}{|c|}{ H3-rules, 2007 edition } \\
\hline & $\begin{array}{c}\text { Class-I } \\
\text { prediction } \\
\mathrm{s} \\
\end{array}$ & \multicolumn{7}{|c|}{ Statistics } \\
\hline & $\begin{array}{c}\text { True } \\
\text { Positives }\end{array}$ & $\begin{array}{c}\text { True } \\
\text { Negatives }\end{array}$ & $\begin{array}{c}\text { False } \\
\text { Positives }\end{array}$ & $\begin{array}{c}\text { False } \\
\text { Negatives }\end{array}$ & Accuracy & Precision & Recall & $\begin{array}{c}\mathrm{F}- \\
\text { measure }\end{array}$ \\
\hline & 178 & 5 & 13 & 20 & 0,85 & 0,93 & 0,90 & 0,92 \\
\hline
\end{tabular}

Table 12: Extended performance measures for Kinked base predictions in CDR-H3 (Phase $1)$. 